\title{
ON THE IRREGULAR CASES OF THE LINEAR ORDINARY DIFFERENCE EQUATION*
}

\author{
BY \\ C. RAYMOND ADAMS
}

Introduction. In the analytic theory of the linear ordinary difference equation

$$
\sum_{i=0}^{n} a_{i}(x) g(x+n-i)=0
$$

whose coefficient functions are rational and expressible in the form

$$
a_{i}(x)=x^{m}\left(a_{i 0}+a_{i 1} x^{-1}+a_{i 2} x^{-2}+\cdots\right) \quad \text { for }|x|>R
$$

and whose characteristic equation is

$$
(i=0,1,2, \cdots, n)
$$

$$
a_{00} \rho^{n}+a_{10} \rho^{n-1}+\cdots+a_{n-1,0} \rho+a_{n 0}=0,
$$

the assumption is commonly made that the roots of (3) are finite, distinct, and different from zero. This narrowly restrictive hypothesis characterizes what we call the regular case. But little attention has yet been devoted to the more general problem of the irregular cases, in which the roots fail to satisfy this hypothesis.

Barnes $\dagger$ in 1905 studied most of the irregular cases of the equation of second order with coefficients linear in $x$, employing the Laplace transformation.

In 1910 Horn $\ddagger$ gave formal series solutions (divergent) and a theorem setting forth the existence of solutions whose asymptotic forms for large real positive values of $x$ are given by the formal series, when the only departure from regularity is the vanishing of one root.

In papers read (by title) before the Society in 1913 Batchelder $\S$ found formal series solutions in all possible irregular cases for the equation of second

\footnotetext{
* Presented to the Society, December 28, 1926; received by the editors in July, 1927.

t On the homogeneous linear difference equation of the second order with linear coefficients, Messenger of Mathematics, vol. 34 (1905), pp. 52-71.

¥Über das Verhalten der Integrale linearer Differenzen- und Differentialgleichungen für grosse Werte der Verdinderlichen, Crelle's Journal, vol. 138 (1910), pp. 159-191, in particular p. 191.

\$ The divergent series satisfying linear difference equations of the second order and The hypergeometric difference equation, Bulletin of the American Mathematical Society, vol. 19 (1912-13), pp. 498, 500-502.
} 
order and sought to extend the theory as developed by Birkhoff* for the regular case to the irregular cases when the coefficient functions are linear in $x$. Batchelder's results are contained in a Dissertation $†$ deposited in the Harvard Library in 1916; they are also incorporated in An Introduction to Linear Difference Equations, presently to be published with the aid of the National Research Council.

Perron + in 1917 derived certain properties of solutions of the equation of the second order whose characteristic equation has equal roots.

The most searching investigation yet made of the irregular cases for the equation of $n$th order is by Galbrun. $\S$ In 1921 he employed the Laplace transformation, as he had done earlier in his study of the regular case,\| in developing the theory of equation (1) when two of the roots of (3) are equal.

Nörlund, in his Differenzenrechnung, does little more than mention the problem of the irregular cases.

The purposes of this paper are first to obtain formal power series solutions of the equation (1) in the irregular cases and secondly to adapt the general methods employed by Birkhoff in the regular case to the problem of finding analytic solutions which shall be asymptotically represented by the formal series. In $\$ 1$ formal series solutions are found for an extensive class of irregular cases. $\$ \S 2,3$, and 4 are devoted to a class of irregular cases which have a theory much like that of the regular case: in $\$ 2$ is proved the existence of two sets of solutions resembling the principal solutions of the regular case; in $\$ 3$ the periodic functions defined by these two sets of solutions are considered briefly; and in $\$ 4$ the asymptotic behavior of these two sets of solutions in the entire plane is examined. A second class of irregular cases is treated in $\$ \$ 5,6,7$, and 8: $\S 5$ is concerned with formal series in the case in which (3) has an $n$-fold root not zero; in $\$ 6$ certain existence theorems are proved in the same case; in $\$ 7$ the results and implications of $\$ \$ 5$ and 6 for $n=2$ are given special attention; and in $\$ 8$ are pointed out certain

* General theory of linear difference equations, these Transactions, vol. 12 (1911), pp. 243-284.

† The hypergeometric difference equation.

‡Über lineare Differenzengleichungen zweiter Ordnung deren charakteristische Gleichung zwei gleiche Wurzeln hat, Sitzungsberichte der Heidelberger Akademie der Wissenschaften (mathematischnaturwissenschaftliche Klasse), vol. 8A (1917), No. 17, 18pp.

Sur certaines solutions exceptionnelles d'une équation linéaire aux différences finies, Bulletin de la Société Mathématique de France, vol. 49 (1921), pp. 276-241.

\|I Sur la représentation des solutions d'une équation linéaire aux différences finies pour les grandes valeurs de la variable, Acta Mathematica, vol. 36 (1913), pp. 1-68.

I Berlin, Springer, 1924, pp. 339-342. 
results which can be inferred for a large class of irregular cases from the study of a particular case made in $\$ \S 5,6$, and 7. Throughout the paper an attempt is made to keep the notation and arrangement parallel to Birkhoff's, and it is assumed that his $t_{2}$ zatment of the regular case is familiar to the reader.

We make the hypothesis that the equation (1) is irreducible; that is, that it is not satisfied by any analytic function of $x$ which is a solution of a difference equation of order less than $n$ with rational coefficients.

1. Formal series solutions. We shall set forth here only a statement of the types of series that formally satisfy (1) in various irregular cases; that these series do satisfy (1) can be verified directly by substitution, although the labor involved is not inconsiderable. The cases in which the only irregularities are multiple roots finite and different from zero are grouped for consideration in

Class $1\left(a_{00} \neq 0, a_{n 0} \neq 0\right)$. Corresponding to a simple root $\rho_{1}$ there is one formal series of the "regular" type:*

$$
s(x)=\rho_{1}^{x} x^{r} P\left(x^{-1}\right) .
$$

Corresponding to a root $\rho_{1}$ of multiplicity $m>1$, and on the assumption that $\rho_{1}$ is not a root of the secondary equation

$$
a_{01} \rho^{n}+a_{11} \rho^{n-1}+\cdots+a_{n-1,1} \rho+a_{n 1}=0,
$$

there are $m$ series of the following type:*

$$
s(x)=\rho_{1}^{x} e^{L^{(m)}(x)} x^{r} P\left(x^{-1 / m}\right),
$$

where

$$
L^{(m)}(x)=\gamma^{(m-1)} x^{(m-1) / m}+\gamma^{(m-2)} x^{(m-2) / m}+\cdots+\gamma^{\prime} x^{1 / m},
$$

the constants $\gamma^{(m-1)}, \gamma^{(m-2)}$, etc., being different in the different series. The $m$ values of $\gamma^{(m-1)}$ are the $m$ determinations of the $m$ th root of a constant not zero times the left-hand member of (5) after $\rho_{1}$ has been substituted for $\rho$,

* $r$ is a constant, in this and subsequent types. $P\left(x^{-1}\right)$ stands for a power series in $x^{-1}$, namely $1+s^{\prime} x^{-1}+s^{\prime \prime} x^{-2}+\cdots$; the constant term may be taken as 1 , since we are dealing with the homogeneous difference equation. Similarly, $P\left(x^{-1 / m}\right)$ is used to represent a power series in $x^{-1 / m}$, the first term being 1 . The $\gamma$ 's, wherever they occur, are constants. In each type of series the constants are calculated (by formal substitution of the series in (1)) in the order in which they appear in the series as written; for example, in (6) the constants after $\rho_{1}$ are calculated in the order $\gamma^{(m-1)}, \gamma^{(m-2)}, \cdots$, $\gamma^{\prime}, r, s^{\prime}, s^{\prime \prime}, \ldots$. The constants thus calculated are uniquely determined for each series of the type. 
and hence are different from zero. It is easily seen that these $m$ series are the $m$ determinations of a single series.*

Corresponding to a root $\rho_{1}$ of multiplicity $m>2$ which is a simple root of the secondary equation (5), there is a set of $m$ series as follows:
1 series,
$s(x)=\rho_{1}^{x} x^{r} P\left(x^{-1 /(m-1)}\right) ;$
$m-1$ series,
$s(x)=\rho_{1}^{x} e^{L^{(m-1)}(x)} x^{r} P\left(x^{-1 /(m-1)}\right)$.

In the case $m=2$, these reduce to "regular" series like (4), but in order that the equation (1) be satisfied by two such series, further conditions must be fulfilled.

When $\rho_{1}$ is an $m$-fold root of the characteristic equation and a multiple root of the secondary equation, additional complications enter the problem of obtaining formal solutions. In general under these conditions we have been able to obtain only part of the full quota of $m$ series; in particular cases the full number has been found, but the facts that the cases are particular and that the statements of conditions are long and involved lead us to omit their description here. Two points may deserve mention: (i) that the presence of $\rho_{1}$ as an $m_{1}$-fold root of the secondary equation tends to reduce to $m-m_{1}$ the index of the root of $x^{-1}$ according to powers of which the series proceed; and (ii) that if $m_{1}$ is sufficiently large, the question of whether $\rho_{1}$ satisfies the subsequent equations

$$
a_{0 j} \rho^{n}+a_{1 j} \rho^{n-1}+\cdots+a_{n-1, j} \rho+a_{n j}=0 \quad(j=2,3, \cdots)
$$

becomes of importance.

We desire to call attention to only one further case. If $\rho_{1}$ is an $m$-fold root of (3), a root of multiplicity $\geqq m$ of (5), a root of multiplicity $\geqq m-1$ of (7) for $j=2$, a root of multiplicity $\geqq m-2$ of (7) for $j=3, \cdots$, a root of multiplicity $\geqq 2$ of (7) for $j=m-1$, and is not a root of (7) for $j=m$, then the equation (1) is satisfied by $m$ series of the regular type (4). In the event of all these hypotheses except the last being satisfied, and if $\rho_{1}$ satisfies all the equations ( 7 ) for $j \geqq m$ (as it would if,-for example, the coefficient functions (2) were polynomials of degree $\leqq m-1$ ), an analytic solution of the equation (1) is $\rho_{1}^{x}$; but this function satisfies a difference equation of first order with rational coefficients, and (1) is therefore reducible.

Secondly we consider the cases in which some or all of the irregularities are due to the presence of zero or infinite roots or of both; these cases we group in

- Cf. $\$ 5$, in which this fact is pointed out more clearly in a particular case. 
Class 2 (one or both of $a_{00}, a_{n 0}=0$ ). Let us denote by $a_{i, j_{i}}$ the first nonzero coefficient in $a_{i}(x)(i=0,1, \cdots, n)$, and choosing $i$ - and $j$-axes, plot the points $\left(i, j_{i}\right)$ as in Figure 1 . Construct a broken line $L$, convex upward, such that both ends of each segment of the line are points of the set $\left(i, j_{i}\right)$ and such that all points of the set lie upon or beneath the line. This is the form that would be assumed by an elastic string if pegs were inserted at the points $\left(i, j_{i}\right)$, the ends of the string fastened one at $\left(0, j_{0}\right)$ and the other at $\left(n, j_{n}\right)$, and the string allowed to contract from above upon the pegs. At least one of the points $\left(i, j_{i}\right)$ will clearly be situated

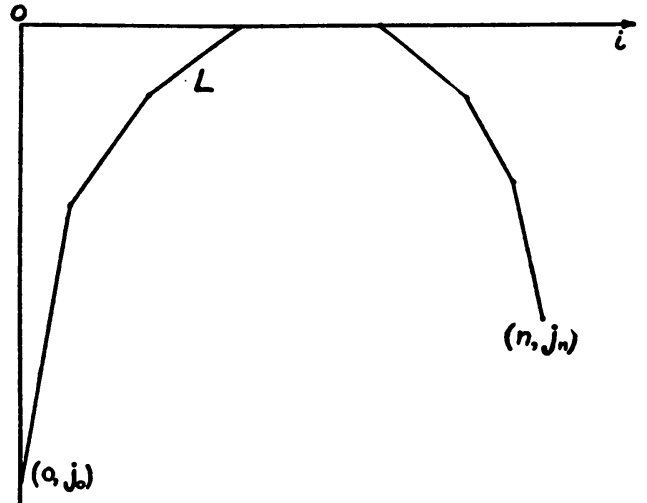
on the $i$-axis; otherwise a power of $x$ might be suppressed in the entire equation (1).

In this class of irregular cases the so-called "characteristic equation" (3) is by no means completely characteristic of the difference equation (1). In fact we would rather say that (3) is replaced by several characteristic equations, one associated with each segment of $L$. The degree of the characteristic equation associated with any segment of $L$ is 1 less than the number of points $\left(i, j_{i}\right)$ that lie on or beneath that segment (inclusive of its end points). The coefficients of this characteristic equation are the $a_{i, j_{i}}$ corresponding to points $\left(i, j_{i}\right)$ actually on that segment of $L$; the coefficient corresponding to a point $\left(i, j_{i}\right)$ beneath the segment is zero. Evidently the sum of the degrees of these several characteristic equations is $n$. If one of the segments is horizontal, the characteristic equation associated with that segment (this may be obtained from (3) by suppressing the zero and infinite roots) picks out its quota of formal solutions precisely as (3) distinguishes $n$ solutions in any case of Class 1. As for a segment not horizontal, let the slope of any such segment be $-\mu$, a rational number different from zero. The transformation*

$$
f(x)=x^{\mu x} e^{-\mu x} g(x)
$$

* In making this transformation one should employ the expansion

$$
\left(1+\frac{i}{x}\right)^{\mu z}=e^{\mu x \log [1+i / x]}=e^{i \mu}\left(1-\frac{i^{2} \mu}{2 x}+\frac{i^{3} \mu(3 i \mu+8)}{24 x^{2}}-\cdots\right) .
$$

The factor $e^{-\mu x}$ is inserted in (8) merely for convenience in annulling the $e^{i \mu}$ here. 
then changes (1) into a new equation of exactly the same type except in the respect that if $-\mu$ is not an integer but a fraction, $q / p$ in lowest terms ( $p$ positive), some of the coefficient functions will be of the form $x^{-s / p} A(x)$, where $s$ is a positive integer and $A(x)$ a power series in $x^{-1}$. The effect of (8) upon the points $\left(i, j_{i}\right)$ is to relocate them in such a way that each segment of the new broken line $L^{\prime}$ that "roofs them over" has a slope $\mu$ greater than that of the corresponding segment of $L$. Thus, in particular, the segment of $L$ whose slope is $-\mu$ becomes a horizontal segment of $L^{\prime}$.

This analysis makes it clear that if $-\mu$ is an integer, the state of affairs with respect to the segment of $L$ having this slope is wholly similar to the situation relative to a horizontal segment and hence analogous to the cases of Class 1. The formal series associated with the segment of slope $-\mu$ are like those enumerated above under Class 1 except for the additional factors $x^{\mu x} e^{-\mu x}$ precedin $\mathrm{e}$ g the power series itself. The equation which plays the rôle of (5) here is th equation whose coefficients are the $a_{i j}$ corresponding to points $(i, j)$ which if plotted in Figure 1 would lie upon a line parallel to this segment of slope $-\mu$ and one unit vertically below it. The tertiary equation for a segment of slope $-\mu$ is one whose coefficients $a_{i j}$ are those whose corresponding points $(i, j)$ if plotted would lie upon a line two units vertically below the segment, and so on for the subsequent equations.

If on the other hand $-\mu$ is a fraction, $q / p$ in lowest terms, there corresponds to each simple root $\rho_{1}$ of the characteristic equation for this segment of $L$ a formal solution of the type

$$
s(x)=x^{\mu x} e^{-\mu x} \rho_{1}^{x} e^{L^{(p)}(x)} x^{r} P\left(x^{-1 / p}\right) .
$$

It is noteworthy that in this case $\gamma^{(p-1)}$, and in addition any or all of the subsequent $\gamma$ 's in the exponent of $e$, may vanish. In fact if all the points $\left(i, j_{i}\right)$ not on the segment of slope $-\mu$ are situated on or beneath a line parallel to this segment and one unit vertically below it, the $\gamma$ 's in (9) will all be zero.

When, for $-\mu=q / p$, the characteristic equation for this segment of $L$ has a root $\rho_{1}$ of multiplicity $m>1$, the situation is more complicated. Let the segment of slope $q / p$ be prolonged in both directions to form a line $l$. Of the points $(i, j)$ corresponding to non-zero coefficients $a_{i j}$ but not situated on this segment there will be one or more whose distance below $l$, measured vertically, is least; let that distance be $t / p$ and draw a line $l^{\prime}$ parallel to $l$ through this point (or these points). Let the constants $a_{i j}$ corresponding to points $(i, j)$ on $l^{\prime}$ be used as coefficients in an algebraic equation of degree $n$; this is the secondary equation for the segment of slope $q / p$. If the secondary equation is not satisfied by $\rho_{1}$ and if $t / m$ is 1 or a submultiple of 1 , then 
corresponding to the root $\rho_{1}$ there are $m$ series of type (9) with $p$ replaced by $w$, where $w=t / m p$. When the secondary equation has $\rho_{1}$ as a root, the situation is analogous to that described in the corresponding case under Class 1 above.

\section{CASES IN WHICH THE THEORY RESEMbLES CLOSELY THAT OF THE REGULAR CASE}

2. Existence theorems. In certain of the irregular cases the theory of equation (1) is much like that of the regular case; these cases fall under the following classes.

Class 2a. The slope of each segment of $L$ is an integer and the characteristic equation associated with each segment has only simple roots.

Class 2b. The slopes of some or all of the segments of $L$ are fractional; the characteristic equation associated with a segment of $L$ whose slope is an integer has only simple roots; the characteristic equation associated with a segment of $L$ whose slope is fractional has only simple roots and either (a) no two of these roots are of equal absolute value, or (b) if two or more of these roots have the same absolute value, no exponential factor $e^{L^{(p)}(x)}$ occurs in the formal series corresponding to them (conditions under which the situation (b) would obtain are described in $\$ 1$ ).

We point out briefly in this section the dissimilarities between the existence theorems in the cases of Class $2 a$ and in the regular case; the discussion requires only slight modifications, chiefly in respect to the formulas, to adapt it to the cases of Class $2 b$.

In each case of Class $2 a$ the equation (1) possesses $n$ formal series solutions which we denote by

$$
s_{i}(x)=x^{\mu_{i} x} e^{-\mu_{i} x} \rho_{i}^{x} x^{r_{i}}\left(1+s_{i}^{\prime} x^{-1}+s_{i}^{\prime \prime} x^{-2}+\cdots\right) \quad(i=1,2, \cdots, n) .
$$

All the $\mu_{i}$ for the set of solutions associated with a particular segment of $L$ then have the same value. To gain the simplicity of the matrix notation and to make our work parallel that of Birkhoff, we write our single equation (1) of the $n$th order in the form of a system of $n$ linear equations of the first order; the $n$ formal solutions (10) then provide us with $n$ sets of formal solutions for the system.* These sets we arrange in a matrix, of which each set constitutes a column; the elements of the first row are the $n$ series (10). The order of the columns in the matrix is of importance. Let them be arranged first according to descending values of $\mu_{i}$; secondly, let those for which $\mu_{i}$ is the same be ordered according to descending values of $\left|\rho_{i}\right|$.

- The details of this frequently employed device are shown fully in a particular case in $\$ 5$. 
Then $(x / e)^{\mu_{i}} \rho_{i}$ plays the rôle that $\rho_{i}$ does in the regular case, and since we have

$$
\left|\left(\frac{x}{e}\right)^{\mu_{i}} \rho_{i}\right| \geqq\left|\left(\frac{x}{e}\right)^{\mu_{j}} \rho_{i}\right|, \quad i>j,
$$

for large values of $|x|$ when $\mu_{i}$ is $>\mu_{j}$ and for all values of $x$ when $\mu_{i}$ is $=\mu_{i}$, no difficulty is experienced in establishing the existence of determinant limits, ${ }^{*}$ of solutions associated with them, and of intermediate solutions.

In seeking solutions by the aid of contour integrals, however, we must in some instances make a different choice of the $\lambda_{j k}$ in Birkhoff's formula (40) in order to insure that $g_{1 k}(x)$ be asymptotically represented by $s_{1 k}(x)$ in as large a region as possible. For $x$ above $A_{1} \infty$ we have, as in the regular case, $\dagger$

$$
\begin{aligned}
g_{1 k}(x) \sim s_{11}(x) e^{2 \pi \lambda_{1 k}(-1)^{1 / 2}} q_{1 k}(x) & +\cdots \\
& +s_{1, k-1}(x) e^{2 \pi \lambda_{k-1, k}(-1)^{1 / 3}} q_{k-1, k}(x)+s_{1 k}(x),
\end{aligned}
$$

and we desire the last term on the right to be the dominant one in the left half-plane. The governing influences in the terms are the exponential factors, which are

$$
\left(\frac{x}{e}\right)^{\mu_{1} x} \rho_{1}^{x} e^{2 \pi \lambda_{1 k}(-1)^{1 / 2} x}, \cdots,\left(\frac{x}{e}\right)^{\mu_{k-1} x} \rho_{k}^{x} e^{2 \pi \lambda_{k-1, k}(-1)^{1 / 2} x},\left(\frac{x}{e}\right)^{\mu_{k} x} \rho_{k}^{x} .
$$

Writing all these as exponentials to the base $e$ and dividing through by the last, we may express the exponents in the following form:

$$
\begin{aligned}
& 2 \pi(-1)^{1 / 2}\left(\lambda_{1 k}-\frac{\mu_{1}-\mu_{k}+\log \rho_{k}-\log \rho_{1}-\left(\mu_{1}-\mu_{k}\right) \log x}{2 \pi(-1)^{1 / 2}}\right) x, \cdots, \\
& 2 \pi(-1)^{1 / 2}\left(\lambda_{k-1, k}-\frac{\mu_{k-1}-\mu_{k}+\log \rho_{k}-\log \rho_{k-1}-\left(\mu_{k-1}-\mu_{k}\right) \log x}{2 \pi(-1)^{1 / 2}}\right) x, 0 .
\end{aligned}
$$

We propose to select the $\lambda$ 's so that these exponents, save the last, will have real parts that become negatively infinite as $x$ becomes infinite in the second quadrant. If the $\mu_{i}(i=1,2, \cdots, k)$ in (13) are all equal, these exponents are identical with the corresponding exponents in the regular case. This makes it clear that the $\lambda$ 's may be chosen so that those solutions on the left,

* We employ the terminology of Birkhoff, loc. cit.

$\dagger$ It should be observed that, to be consistent with the definition of asymptotic representation of a function $g(x)$ (cf. Birkhoff, loc. cit., p. 248), the relation (12) should, until the question of dominance is settled, be interpreted as meaning " $g_{1 k}(x)$ is the sum of $k$ functions which have for asymptotic forms the several terms on the right." For simplicity, however, we shall continue to write such relations in the form of (12). 
obtained by contour integrals, which are associated with the segment of $L$ farthest to the left will have the same properties as do all the principal solutions in the regular case. When $\mu_{i}$ is thus equal to $\mu_{k}$, we choose $\lambda_{i k}$ as the least integer exceeding*

$$
\frac{\arg \rho_{k}-\arg \rho_{i}}{2 \pi} .
$$

When $\mu_{i}$ is greater than $\mu_{k}$, the $i$ th exponent in (13) clearly has a real part that becomes negatively infinite as $x$ recedes to infinity in the sector $\pi / 2+\epsilon \leqq \arg x<\pi, \epsilon$ being an arbitrarily small positive number, whatever choice of $\lambda_{i k}$ be made; this is owing to the presence of $\log x$ within the parentheses. If, however, we choose $\lambda_{i k}$ to be any integer (and we take it to be the least integer) greater than

$$
\alpha_{i k}=\frac{\arg \rho_{k}-\arg \rho_{i}-\left(\mu_{i}-\mu_{k}\right) \pi / 2}{2 \pi}
$$

the asymptotic form of $g_{1 k}(x)$ will be given by $s_{1 k}(x)$ for $x$ above $A_{1} w$ and on or to the left of the imaginary axis, or likewise on or to the left of any parallel to it.

For $x$ in the strip bounded by $A_{1} \infty$ and $B_{1} \infty$ the asymptotic form of $g_{1 k}(x)$ is given by $s_{1 k}(x)$ as in the regular case, by virtue of the ordering of the formal series according to $\mu$ 's and $\rho$ 's.

When $x$ is below $B_{1} \infty$ we have

$$
\begin{aligned}
& g_{1 k}(x) \sim s_{11}(x) e^{2 \pi\left(\lambda_{1 k-1}\right)(-1)^{1 / 2}} q_{1 k}(x)+\cdots \\
& +s_{k-1, k}(x) e^{2 \pi\left(\lambda_{k-1, k-1)(-1)^{1 / 2}} x\right.} q_{k-1, k}(x)+s_{1 k}(x) .
\end{aligned}
$$

The dominance depends upon the real part of the exponents

$$
\begin{aligned}
& 2 \pi(-1)^{1 / 2}\left(\lambda_{1 k}-1-\frac{\mu_{1}-\mu_{k}+\log \rho_{k}-\log \rho_{1}-\left(\mu_{1}-\mu_{k}\right) \log x}{2 \pi(-1)^{1 / 2}}\right) x, \cdots, \\
& 2 \pi(-1)^{1 / 2}\left(\lambda_{k-1, k}-1-\frac{\mu_{k-1}-\mu_{k}+\log \rho_{k}-\log \rho_{k-1}-\left(\mu_{k-1}-\mu_{k}\right) \log x}{2 \pi(-1)^{1 / 2}}\right) x, 0 .
\end{aligned}
$$

For $\mu_{i}=\mu_{k}(i=1,2, \cdots, k-1)$ the asymptotic form of $g_{1 k}(x)$ in any sector $\pi<\arg x \leqq 3 \pi / 2-\epsilon$ for which $\epsilon$ is positive is $s_{1 k}(x)$. If none of the quantities

* This choice is the same as Birkhoff's in the regular case when the quantity (14) is not an integer; if (14) is an integer, our $\lambda_{i k}$ exceeds his by 1 . We make this choice in order to insure that $\boldsymbol{g}_{1 k}(x)$ always be represented asymptotically by $s_{1 k}(x)$ in the direction of the positive axis of imaginaries; with Birkhoff's choice and (14) an integer for one or more values of $i$, the asymptotic form of $g_{1 k}(x)$ in that direction is given by the sum of two or more terms of (12), one of which is the last. 
(14) is an integer, we have $g_{1 k}(x) \sim s_{1 k}(x)$ for $\pi<\arg x \leqq 3 \pi / 2$; if on the other hand one or more of the quantities (14) is an integer, the asymptotic form of $g_{1 k}(x)$ in the direction of the negative axis of imaginaries is given by the sum of two or more terms of (16), one of which is the last.

When $\mu_{i}$ is greater than $\mu_{k}$ for some or all of the values of $i(=1,2, \cdots$, $k-1)$, the $i$ th exponent in (17) has a real part that becomes negatively infinite as $x$ recedes to infinity in the sector $\pi<\arg x \leqq 3 \pi / 2-\epsilon$, due to the presence of the term $\log x$, so that we have $g_{1 k}(x) \sim s_{1 k}(x)$ in that sector. In the direction of the negative axis of imaginaries, however, the asymptotic form of $g_{1 k}(x)$ is given by $s_{1 k}(x)$ only when all of the following conditions are fulfilled: (a) $\mu_{i}-\mu_{k}$ is $\leqq 1$ for $i=1,2, \cdots, k-1$; (b) when $\mu_{i}-\mu_{k}=1$, $\lambda_{i k}-\alpha_{i k}$ is $<\frac{1}{2}$; (c) when $\mu_{i}-\mu_{k}$ is 0 , the quantity (14) is not an integer. If among these conditions (c) alone fails to be satisfied, the asymptotic form of $s_{1 k}(x)$ in the direction of the negative axis of imaginaries is given by the sum of two or more terms of (16); one of these terms is the last, while the others correspond to values of $i$ for which $\mu_{i}=\mu_{k}$ and (14) is an integer. If either or both of conditions (a) and (b) fail, the asymptotic form of $s_{1 k}(x)$ in the direction in question is given by the term (or sum of terms) of (16) corresponding to the value (or values) of $i$ for which

$$
\lambda_{i k}-1-\alpha_{i k}+\frac{\mu_{i}-\mu_{k}}{2}
$$

is largest.

The functions $g_{i j}(x)$ are analytic except for poles throughout the entire finite plane; we denote the matrix $\left(g_{i j}(x)\right)$ by $G(x)$.

The freedom that we have in the choice of $\lambda_{i k}$ when $\mu_{i}$ is $>\mu_{k}$ makes it clear that the solutions on the left associated with segments of $L$ other than that farthest to the left are not in general characterized uniquely by the properties we have proved for them. Exceptions can occur only when the solutions in question are associated with the second segment from the left and when the slope of that segment is only 1 greater than the slope of the first segment. We therefore hesitate to apply to these solutions the term "principal solutions" except in the case in which $L$ consists of but a single segment; this case is, however, essentially regular.

There exists a similar set of solutions "on the right," in obtaining which we choose $\lambda_{i k}(i>k)$ as the least integer exceeding $\alpha_{i k}$ (cf. (15); the fact that in $\alpha_{i k}, i$ is now $>k$ should not be overlooked). From the relation $\alpha_{i k}=-\alpha_{k i}$ it follows that $\lambda_{k i}=2-\lambda_{i k}$ or $1-\lambda_{i k}$ according as $\alpha_{i k}$ is or is not an integer. In this set of solutions only those associated with the segment of $L$ farthest to the right can in general be said to be characterized by the properties we prove them to possess. We denote the matrix of these functions by $H(x)$; 
its elements are analytic except for poles throughout the finite plane.

3. Periodic functions. We shall examine briefly the matrix of periodic functions $P(x)$ defined by

$$
G(x)=H(x) P(x) ;
$$

that is,

$$
P(x)=H^{-1}(x) G(x) .
$$

The elements of $H^{-1}(x)$ and $G(x)$ are analytic except for poles over the entire finite plane. Hence in any period strip the elements $p_{i j}(x)$ of $P(x)$ are analytic except for poles throughout the finite portion of the strip. We set $z=e^{2 \pi(-1)^{1 / 2} x}$ and regard $p_{i j}(x)$ as a function of $z$; the upper end of the strip corresponds to $z=0$, the lower end to $z=\infty$.

At the upper end of the strip we have

$$
P(x) \sim S^{-1}(x) S(x),
$$

where $S(x)$ is the matrix $\left(s_{i j}(x)\right)$ and $s_{i j}(x)$ is $s_{i}(x+i-1)$; this gives us the asymptotic relation

$$
p_{i j}(x) \sim\left(\frac{x}{e}\right)^{\left(\mu_{\left.j-\mu_{i}\right) x}\right.}\left(\frac{\rho_{j}}{\rho_{i}}\right)^{x} x^{r_{j}-r_{i} \delta_{i j},} \quad \delta_{i j}=\left\{\begin{array}{l}
1, i=j ; \\
0, i \neq j .
\end{array}\right.
$$

It follows that for $x$ sufficiently high up in the strip we have

$$
p_{i j}(x)=e^{2 \pi \lambda_{i j}(-1)^{1 / 2} x}\left(c_{i j}+c_{i j}^{\prime} e^{2 \pi(-1)^{1 / 2} x}+\cdots\right),
$$

where $\lambda_{i i}$ is defined to be zero and $c_{i i}$ is 1 ; the series in parentheses is convergent in the vicinity of $z=0$.

At the lower end of the strip we have a situation which in form, though not in substance, is much more complicated than the one we meet in the regular case. The asymptotic form of $G(x)$ is not in general $S(x)$, nor is the asymptotic form of $H^{-1}(x)$ given by the product of $S^{-1}(x)$ and a simple matrix. An examination of $p_{i j}(x)$ readily shows that it is analytic or becomes infinite to only a finite order at $z=\infty$, but the determination of that order explicitly in terms of the $\mu$ 's and $\rho$ 's is not feasible.* We conclude that the

* Such a determination might be made feasible by reassigning the values of the $\lambda$ 's in (12) when $\mu_{1}$ is $>\mu_{k}$ [and in the similar expression for the form of $h_{1 k}(x)$ in the first quadrant above $A_{1} \infty$ when $\mu_{n}$ is $\left\langle\mu_{k}\right.$ ] so as to insure that $g_{1 k}(x)$ be given asymptotically, as $x$ becomes infinite along a parallel to the negative axis of imaginaries, by, for example,

where

$$
s_{11}(x) e^{X} q_{1 k}(x) \quad\left[s_{1 n}(x) e^{Y} q_{1^{k}}^{\prime}(x)\right],
$$

$$
X=2 \pi\left(\lambda_{1 k}-1\right)(-1)^{1 / 2} x, \quad Y=2 \pi\left(\lambda_{n k}-1\right)(-1)^{1 / 2} x,
$$

and in addition by imposing the restriction that for all values of $k$ associated with the first segment of $L$ on the left [last segment of $L$ on the right] the expression (14) be not an integer. This restriction, if we take for $\arg \rho_{i}(i=1,2, \cdots, n)$ the principal values of the arguments, means that no two of the roots of the characteristic equation associated with the first segment of $L$ on the left [last segment of $L$ on the right] may have the same argument. 
elements $p_{i j}(x)$ are rational functions of $e^{2 \pi(-1)^{1 / 2}}$. Let the order of the pole of $p_{i j}(x)$ at $\infty$ be denoted by $\nu_{i j}$; then for values of $x$ sufficiently low in the strip we have

$$
p_{i j}(x)=e^{2 \pi r_{i j}(-1)^{1 / 2} x}\left(d_{i j}+d_{i j}^{\prime} e^{-2 \pi(-1)^{1 / 2}} x+\cdots\right) .
$$

It may be remarked that if the coefficient functions of our system of equations have no finite poles, as is the case when $a_{0}(x)$ reduces to a constant, then the elements of $G(x)$ are analytic without exception over the finite plane and the series in parentheses in (19) are polynomials. Similarly, if the inverse of the matrix of these coefficient functions has no finite poles, and it will have nore if $a_{n}(x)$ is a constant, the elements of $H(x)$ have no poles in the finite plane and $P^{-1}(x)$ is a matrix of functions each of which is a polynomial in $z$ multiplied by some power of $z$. Except in respect to the formulas this section needs practically no modification to adapt it to the cases of Class $2 \mathrm{~b}, \S 2$.

4. The solutions $G(x)$ and $H(x)$ in the entire plane. We are now in a position to examine the asymptotic form of $g_{i j}(x)$ (or $\left.h_{i j}(x)\right)$ in the entire plane. The equation (18) gives us

$$
g_{i j}(x)=h_{i 1}(x) p_{1 j}(x)+\cdots+h_{i n}(x) p_{n j}(x) .
$$

Along a ray from the origin in the first quadrant above the axis of reals we have

and therefore

$$
p_{i j}(x) \sim e^{2 \pi \lambda_{i j}(-1)^{1 / 2}{ }^{x}} c_{i j}
$$

$$
g_{i j}(x) \sim s_{i 1}(x) e^{2 \pi \lambda_{1 j}(-1)^{1 / 2} x} c_{1 i}+\cdots+s_{i n}(x) e^{2 \pi \lambda_{n j}(-1)^{1 / 2}} c_{n j} .
$$

The question of which term dominates turns upon the relative magnitude of the absolute values of

$$
\begin{aligned}
& \left(\frac{x}{e}\right)^{\mu_{1} x} \rho_{1}^{x} e^{2 \pi \lambda_{1 j}(-1)^{1 / 2} x}, \cdots,\left(\frac{x}{e}\right)^{\mu_{j} x} \rho_{j}^{x}, \cdots, \\
& \left(\frac{x}{e}\right)^{\mu_{n} x} \rho_{n}^{x} e^{2 \pi \lambda_{n j}(-1)^{1 / 2} x} .
\end{aligned}
$$

Dividing by $(x / e)^{\mu_{j}{ }^{x} \rho_{j}{ }^{x}}$ and expressing the factors all in the form of exponentials to the base $e$, we find the exponents to be as follows:

$$
\begin{aligned}
& 2 \pi(-1)^{1 / 2}\left(\lambda_{1 j}-\frac{\mu_{1}-\mu_{j}+\log \rho_{j}-\log \rho_{1}-\left(\mu_{1}-\mu_{j}\right) \log x}{2 \pi(-1)^{1 / 2}}\right) x, \cdots, 0, \cdots, \\
& 2 \pi(-1)^{1 / 2}\left(\lambda_{n j}-\frac{\mu_{n}-\mu_{j}+\log \rho_{j}-\log \rho_{n}-\left(\mu_{n}-\mu_{j}\right) \log x}{2 \pi(-1)^{1 / 2}}\right) x .
\end{aligned}
$$


We discuss separately the cases in which $j$ is associated with the segment of $L$ farthest to the left and the cases in which it is not. Let us begin with the first of these cases.

For values of $i$ not associated with this first segment of $L, \mu_{i}$ is $<\mu_{j}$ and the exponent

$$
2 \pi(-1)^{1 / 2}\left(\lambda_{i j}-\frac{\mu_{i}-\mu_{j}+\log \rho_{j}-\log \rho_{i}-\left(\mu_{i}-\mu_{j}\right) \log x}{2 \pi(-1)^{1 / 2}}\right) x
$$

has a real part which becomes negatively infinite as $x$ recedes to $\infty$ in any manner whatever in the sector $0 \leqq \arg x \leqq \pi / 2$. The terms of (20) for these values of $i$ can therefore play no part in the asymptotic form of $g_{i j}(x)$ in the region under consideration. For values of $i$ associated with this segment of $L, \mu_{i}=\mu_{j}$ and the coefficient of $x$ in the $i$ th exponent of the set (21) is a constant. Let the points $P_{i}(i=1,2, \cdots, m)$ represent these coefficients in the complex plane; by virtue of our definition of $\lambda_{i j}$ all these points except $P_{j}$, which is at the origin, lie above the axis of reals and below or upon the line $v=2 \pi$. Furthermore, by the ordering of the $\rho_{i}$ the points of $P_{i}$ proceed from right to left as $i$ increases. To allow $x$ to become infinite along a ray $\arg x=\tau$ is precisely the same thing, so far as the behavior of the first $m$ exponents in (21) is concerned, as to rotate the whole set of points $P_{i}$ bodily through the angle $\tau$ and then allow $x$ to become infinite along the positive axis of reals; that point of the set which in the rotated position lies farthest to the right corresponds to the dominant term. Let us construct, then, the broken line $P_{1}, \cdots, P_{j}, \cdots, P_{m}$ above or upon which all the points $P_{i}$ lie and the vertices of which are points of the set. We are interested in what happens as $x$ becomes infinite in the first quadrant; i.e., in values of $\tau$ between 0 and $\pi / 2$. The dominant terms correspond, therefore, to the points $P_{i}$ which are vertices of the broken line $P_{1}, \cdots, P_{m}$. Let the acute angles which the successive segments of the portion $P_{1}, \cdots, P_{j}$ of this broken line, as we proceed from right to left, make with the axis of reals be denoted by $\phi_{1}, \phi_{2}, \cdots$. Then the rays

$$
\frac{\pi}{2}-\phi_{1}, \frac{\pi}{2}-\phi_{2}, \cdots
$$

are, in increasing angular order, the critical rays along each of which the asymptotic form of $g_{i j}(x)$ changes uniformly in the sense of Birkhoff; i.e., along the ray arg $x=\pi / 2-\phi_{\alpha}$, the form changes uniformly from

$$
s_{i r}(x) e^{2 \pi \lambda_{r j}(-1)^{1 / 2} x} c_{r j} \quad \text { to } s_{i t}(x) e^{2 \pi \lambda_{t j}(-1)^{1 / 2} x} c_{t j} .
$$

Thus the behavior of the solutions associated with the segment of $L$ farthest 
to the left is wholly analogous to the behavior of all the solutions in the regular case.

We turn our attention now to the case in which $j$ is not associated with the segment of $L$ farthest to the left. From (22) it is clear that as $x$ becomes infinite in any manner whatsoever in the sector $0 \leqq \arg x \leqq \pi / 2-\epsilon$, the terms corresponding to values of $i$ that belong to the first segment of $L$ on the left dominate all other terms. If there is only one solution associated with that segment of $L$, it follows therefore that we have

$$
g_{i j}(x) \sim s_{i 1}(x) e^{2 \pi \lambda_{1 j}(-1)^{1 / 2} x} c_{1 j} \quad(j=2,3, \cdots, n)
$$

in the sector $\epsilon \leqq \arg x \leqq \pi / 2-\epsilon$. If there are several solutions, corresponding to $i=1,2, \cdots, m$, associated with that segment, we must determine which one dominates. The real part of (22) may be written (setting $x=u+i v$; $u, v$ real):

$$
\begin{aligned}
f_{i}(u, v)=u \log \left|\rho_{i}\right| & +u\left[\left(\mu_{i}-\mu_{j}\right)(\log |x|-1)-\log \left|\rho_{i}\right|\right] \\
& -2 \pi \lambda_{i j} v-v \arg \rho_{i}+v\left[\arg \rho_{j}-\left(\mu_{i}-\mu_{j}\right) \arg x\right]
\end{aligned}
$$

The quantities in brackets are the same for $i=1,2, \cdots, m$; hence along any particular ray $\arg x=\tau$, or $u=s v$, within the first quadrant that term will dominate for which

$$
s \log \left|\rho_{i}\right|-2 \pi \lambda_{i j}-\arg \rho_{i}
$$

is largest. For simplicity let us set

and

$$
\lambda_{i j}-\alpha_{i j}=a_{i j},
$$

$$
b_{k l}=\frac{2 \pi\left(\lambda_{k j}-\lambda_{l j}\right)+\arg \left(\rho_{k} / \rho_{l}\right)}{\log \left|\rho_{l} / \rho_{k}\right|}=\frac{2 \pi\left(a_{k j}-a_{l_{j}}\right)}{\log \left|\rho_{l} / \rho_{k}\right|} ;
$$

we observe $b_{k l}=b_{l k}$. Then it follows from the ordering of the $\rho_{i}$ according to descending absolute value that the first term of (20) dominates when $s$ satisfies all the inequalities

$$
s>b_{1 k} \quad(k=2,3, \cdots, m) .
$$

Secondly, if $s$ satisfies the conditions

$$
s<b_{12} \text { and } s>b_{2 k} \quad(k=3,4, \cdots, m),
$$

the second term of (20) dominates. Thus we see that if any of the quantities $b_{2 k}$ are $\geqq b_{12}$, there is no region in which the second term dominates. Again, if we have

$$
s<b_{13}, s<b_{23} \text {, and } s>b_{8 k} \quad(k=4,5, \cdots, m),
$$


the third term dominates; and so on. Finally, if we have

$$
s<b_{m k} \quad(k=1,2, \cdots, m-1),
$$

the $m$ th term dominates. It is clear that if any of the $b_{m k}$ are $\leqq 0$, the $m$ th term cannot dominate along any ray in the first quadrant. The rays determined by setting $s$ successively equal to

$$
b_{12}, b_{13}, b_{23} \text {, etc. }
$$

constitute a set of rays of which some may be critical; along one of these critical rays, if there are any, the asymptotic form of $g_{i j}(x)$ changes uniformly from one of the first $m$ terms of (20) to another. Any one of these rays may not be critical in a given instance; or it may happen in a given case that the $b_{1 k}(k=2,3, \cdots, m)$ are all negative or zero, so that the first term dominates along any ray in the first quadrant (exclusive of its boundaries) and there are no critical rays in the quadrant. It is clear that in any event the number of critical rays is $\leqq m-1$.

The asymptotic form of $g_{i j}(x)$ along any ray $R$ through the origin within the first quadrant and above the uppermost critical ray, however close $R$ may be to the positive $v$-axis but different from it, is given by one of the first $m$ terms of (20). Yet along that axis itself we have $g_{i j}(x) \sim s_{i j}(x)$. How does the form change from $s_{i j}(x)$ to the other term of $(20)$ ? We now investigate this question.

As $x$ becomes infinite along any ray through the origin inclined only slightly to the right of the positive $v$-axis, the quantities (22) for which $\mu_{i}>\mu_{j}$ have real parts all of which are positive for $x$ sufficiently far out on this ray, whereas on the axis itself these real parts are all negative, by our choice of $\lambda_{i j}$. This indicates that for each value of $i$ for which $\mu_{i}>\mu_{j}$ there is a curve $C_{i}$ which as it recedes from the origin ultimately lies in any preassigned sector $\pi / 2-\epsilon \leqq \arg x \leqq \pi / 2$, however small $\epsilon$ may be, and to the left of which (23) is negative and to the right of which (23) is positive. The equation of $C_{i}$ is of course $f_{i}(u, v)=0$. The fact that along any ray in the first quadrant not parallel to the $v$-axis $f_{i}(u, v)$ is ultimately positive for the values of $i$ in question, while along any ray in that quadrant parallel to the $v$-axis $f_{i}(u, v)$ is ultimately negative, shows that the curve $C_{i}$ has no rectilinear asymptote.

To examine further the character of $C_{i}$ let us seek its intersection with the line $u=s v(s>0)$. If we solve for $\log |x|$ and make use of the relation

we find

$$
2 \pi \lambda_{i j}+\arg \rho_{i}-\arg \rho_{i}=2 \pi a_{i j}-\left(\mu_{i}-\mu_{i}\right) \pi / 2,
$$




$$
\log |x|=\log \left|\frac{\rho_{j}}{\rho_{i}}\right|^{1 /\left(\mu_{i}-\mu_{j}\right)}+\frac{2 \pi a_{i j}}{s\left(\mu_{i}-\mu_{j}\right)}+1-\frac{\pi / 2-\arg x}{s} .
$$

Inasmuch as $s$ is the tangent of $\pi / 2-\arg x$, we see that as $s$ approaches zero and $\arg x$ approaches $\pi / 2, \log |x|$ and therefore $|x|$ increases indefinitely. Moreover $(\pi / 2-\arg x) / s$ approaches 1 , so that $\log |x|$ is asymptotic to the sum of the first two terms on the right in (24). In other words, the curve $C_{i}$ is asymptotic to the following curve in which $(r, \theta)$ are the polar coördinates of $x$, the positive $v$-axis being taken as the polar axis with the pole at the origin:

$$
r=\left|\frac{\rho_{j}}{\rho_{i}}\right|^{1 /\left(\mu_{i}-\mu_{j}\right)} e^{-2 \pi a_{i j} /\left(\left(\mu_{i}-\mu_{j}\right) \tan \theta\right)} .
$$

Differentiating (23) we find the slope of $C_{i}$ to be

$$
\frac{d v}{d u}=\frac{\left(\mu_{i}-\mu_{j}\right) \log \left(u^{2}+v^{2}\right)^{1 / 2}+\log \left|\rho_{i} / \rho_{j}\right|}{2 \pi a_{i j}+\left(\mu_{i}-\mu_{j}\right)\left(\tan ^{-1}(v / u)-\pi / 2\right)}
$$

which is positive for values of $\tan ^{-1}(v / u)$ only slightly less than $\pi / 2$; hence the curve $C_{i}$, after it has receded from the origin a sufficient distance, rises monotonically toward the right.

Of the curvilinear asymptotes (25) that which, in a region sufficiently remote from the origin, lies farthest to the left is given by the value of $i$ for which

$$
\frac{2 \pi a_{i j}}{\mu_{i}-\mu_{i}}
$$

is least; we assume for the present that this quotient takes on its minimum for only one value of $i$ and call this value $k$. The order of the curves $C_{i}$ from left to right is according to increasing values of this quotient.

We propose to examine the behavior of $f_{i}(u, v)$ as $x$ becomes infinite along certain curves of the type of (25); i.e., along the curves $r=e^{-l / \theta}(l>0)$. Changing the variables $(u, v)$ to the polar coördinates $(r, \theta)$, we find

$$
\begin{gathered}
f_{i}(u, v)=\phi_{i}(r, \theta)=r\left(\mu_{j}-\mu_{i}\right) \sin \theta\left\{\log \left[r+\left|\frac{\rho_{j}}{\rho_{i}}\right|^{1 /\left(\mu_{i}-\mu_{j}\right)} e^{-2 \pi a_{i j} /\left(\left(\mu_{i}-\mu_{j}\right) \tan \theta\right)}\right]\right. \\
\left.+\frac{\theta}{\tan \theta}-1\right\} .
\end{gathered}
$$

First let $l$ be any positive constant less than the smallest of the quotients (26). Then the curve $r=e^{-l / \theta}$ lies to the left of all the curves $C_{i}$ and as $x$ 
becomes infinite along this curve, or as $\theta$ approaches zero through negative values, we have

$$
\phi_{i}(r, \theta)=\phi_{i}\left(e^{-l / \theta}, \theta\right) \rightarrow e^{-l / \theta}\left(\mu_{i}-\mu_{j}\right)\left(l-\frac{2 \pi a_{i j}}{\mu_{i}-\mu_{j}}\right) .
$$

Thus we see that along any such curve the real part of the exponent (22), for values of $i$ in which we are interested, becomes negatively infinite, and the asymptotic form of $g_{i j}(x)$ is $s_{i j}(x)$.

If $l$ be taken as any constant greater than the least of the quantities (26) but less than the next larger one, it is clear that along the curve $r=e^{-l / \theta}$, the real part of the exponent (22) given by the value $i=k$ becomes positively infinite, whereas all the others become negatively infinite in real part. Thus along such a curve the asymptotic form of $g_{i j}(x)$ is given by the $k$ th term of (20). If $l$ be taken larger than the next to the smallest quotient (26), two or more of the exponents (22) have real parts that become positively infinite along this curve and we have to determine their relative magnitudes. To this end consider the difference $f_{k}(u, v)-f_{i}(u, v)$; for values of $i$ for which $\mu_{k}=\mu_{i}$, this reduces to

$$
u \log \left|\frac{\rho_{k}}{\rho_{i}}\right|-2 \pi v\left(a_{k j}-a_{i j}\right) .
$$

Along any curve for which $v / u$ becomes infinite, such as $r=e^{-l / \theta}$, the second term in (27) dominates. But since $a_{k j}-a_{i j}<0$ when $\mu_{k}=\mu_{i}$, this term is positive and $f_{k}(u, v)$ dominates $f_{i}(u, v)$. Secondly for values of $i$ for which $\mu_{k} \neq \mu_{i}$, we change the variables to $(r, \theta)$ and find

$$
\begin{gathered}
f_{k}(u, v)-f_{i}(u, v)=\phi_{k}(r, \theta)-\phi_{i}(r, \theta) \\
=r\left(\mu_{i}-\mu_{k}\right) \sin \theta\left\{\log \left[r \div\left|\frac{\rho_{k}}{\rho_{i}}\right|^{1 /\left(\mu_{i}-\mu_{k}\right)} e^{2 \pi\left(a_{h j}-a_{i j}\right) /\left(\left(\mu_{i}-\mu_{k}\right) \tan \theta\right)}\right]+\frac{\theta}{\tan \theta}-1\right\} .
\end{gathered}
$$

As $x$ becomes infinite along the curve $r=e^{-l / \theta}$, we have

$$
f_{k}(u, v)-f_{i}(u, v) \rightarrow e^{-l / \theta}\left(\mu_{k}-\mu_{i}\right)\left(l-\frac{2 \pi\left(a_{k j}-a_{i j}\right)}{\mu_{k}-\mu_{i}}\right) .
$$

When $\mu_{i}<\mu_{k}, f_{k}(u, v)$ dominates $f_{i}(u, v)$ for $l$ greater than

$$
\frac{2 \pi a_{k j}}{\mu_{k}-\mu_{j}}
$$

since this ratio is now greater than 


$$
\frac{2 \pi\left(a_{k j}-a_{i j}\right)}{\mu_{k}-\mu_{i}} .
$$

When $\mu_{i}>\mu_{k}$, (29) is less than (30), and for values of $l$ between the two, $f_{k}(u, v)$ dominates $f_{i}(u, v)$.

The above analysis makes it clear that along the curve $C_{i}$ farthest to the left the asymptotic form of $g_{i j}(x)$ shifts from $s_{i j}(x)$ to the $k$ th term of (20). When $\mu_{k}$ is the slope of the segment of $L$ farthest to the left, the new asymptotic form is valid out to the uppermost critical ray, for the asymptotic form cannot shift to a term of (20) associated with another segment of $L$ than the one farthest to the left and it can shift to another term of (20) associated with the same segment of $L$ only along a certain ray, whose equation is obtained by setting (27) equal to zero. If $\mu_{k}$ is not the slope of the first segment of $L$ on the left, the new form holds to the right as far as the curve

$$
r=e^{-2 \pi\left(a_{k j}-a_{p j}\right) /\left(\left(\mu_{k}-\mu_{p}\right) \theta\right)},
$$

where $p$ is the value of $i$ for which (30) takes on its least value exceeding (29). Along this curve the form changes to the $p$ th term of (20). If $p$ is associated with the segment of $L$ farthest to the left, the present asymptotic form holds clear out to the uppermost critical ray; otherwise, it is valid to the right as far as another critical curve (of the same type as (31)) where a further change takes place, and so on. Each time the form changes, as we go toward the right, the new asymptotic form is a term of (20) associated with a segment of $L$ to the left of that to which the former dominant term belonged. We conclude, therefore, that the number of critical curves is at least 1 and is less than or equal to the number of segments of $L$ lying to the left of that with which $j$ is associated.

In the event of the quotient (26) taking on its minimum value for two values of $i$, say $k$ and $k^{\prime}$, we may ascertain which term dominates by inspection of (27) or (28). If $\mu_{k}=\mu_{k^{\prime}},(27)$ shows that the term for which $\left|\rho_{i}\right|$ is greater is the dominating one. It should be observed that $\left|\rho_{k}\right|$ and $\left|\rho_{k^{\prime}}\right|$ cannot be equal, for in the case in question $a_{k j}$ and $a_{k^{\prime} j}$ are the same; hence by definition of the $\lambda_{i j}$, we have $\arg \rho_{k}=\arg \rho_{k^{\prime}}$, and if the absolute values of these $\rho$ 's were also equal, we should have a multiple root, which is contrary to our hypotheses. If $\mu_{k} \neq \mu_{k^{\prime}}$, (28) shows that the dominating term is the one for which $\mu_{i}$ is greater.

Since the definition of asymptotic form is commonly given for a region,* it may be well to make a statement in terms of sectors concerning the asymp-

* Cf Birkhoff, loc. cit., p. 248. 
totic form of $g_{i j}(x)$ in the first quadrant. Denoting by $\arg x=\alpha, \arg x=\beta$, $\cdots, \arg x=\gamma$ the critical rays in increasing angular order, we have

$$
g_{i j}(x) \sim s_{i p}(x) e^{2 \pi \lambda_{p j}(-1)^{1 / 2} x}\left\{\begin{array}{l}
p=r \text { for } \epsilon \leqq \arg x \leqq \alpha-\epsilon ; \\
p=t \text { for } \alpha+\epsilon \leqq \arg x \leqq \beta-\epsilon ; \\
\dot{p}=\dot{w} \text { for } \gamma+\epsilon \leqq \arg x \leqq \pi / 2-\epsilon
\end{array}\right.
$$

where $\epsilon$ is arbitrarily small and positive.

A detailed study of the asymptotic form of $g_{i j}(x)$ in the sector $3 \pi / 2-\epsilon$ $\leqq$ arg $x<2 \pi$ lacks interest because in the last section it was not feasible to determine the $\nu_{i j}$ in terms of the $\mu$ 's and $\rho$ 's and because there are no fundamental differences between the situations in this region and in the first quadrant. We merely remark that there are, in general, both critical rays and critical curves along which the form of $g_{i j}(x)$ changes from one to another of the terms of the sum

$s_{i j}(x) e^{2 \pi\left(v_{i j}-\mu_{1}\right)(-1)^{1 / 2}} e^{-2 \pi r_{1}(-1)^{1 / 2}} d_{1 j}+\cdots+s_{i n}(x)^{2 \pi\left(v_{n i}-\mu_{n}\right)(-1)^{1 / 2} x} e^{-2 \pi r_{n}(-1)^{1 / 2}} d_{n i}$,

the $s_{i j}(x)(j=1,2, \cdots, n)$ standing for the same determination of the series here as in (20).

In the cases of Class $2 \mathrm{~b}, \S 2$, the series associated with segments of $L$ having fractional slopes may contain no exponential factors $e^{L^{(p)}(x)}$. If such is the case, the work of this section (as well as that of $\S \S 2$ and 3) is unchanged save for the fact that it must be borne in mind that some or all of the $\mu$ 's are fractional. If exponential factors do occur, but if none are present in the series corresponding to the segment of $L$ farthest to the left, the situation as regards critical rays is no different from that described above. If exponential factors occur in the series associated with the segment of $L$ farthest to the left, then each critical ray is in general replaced by a critical curve which, as it recedes from the origin, ultimately lies in any given small sector enclosing the ray which, were it not for the presence of the exponential factors, would be critical; these critical curves are, however, algebraic rather than transcendental. If exponential factors occur in the series associated with the segment of $L$ to which $j$ belongs or with a segment to the left of it, the position and nature of these critical curves lying in the sector $\pi / 2-\epsilon$ $\leqq \arg x \leqq \pi / 2$ are in general altered; the investigation of the precise nature of the alteration involves considerable algebraic difficulties and we shall not pause to go into it. 
CASES IN WHICH SOME OR ALL OF THE CHARACTERISTIC EQUATIONS HAVE MULTIPLE ROOTS

Of the cases in this class that in which the equation (3) has $n$ equal roots, finite and different from zero and not satisfying the secondary equation, is to a large extent typical. The next three sections are devoted to the development of existence theorems for this case; the discussion is followed by a section describing the respects in which this case exemplifies the cases in which roots of different multiplicities occur and pointing out the nature of the existence theorems which can be proved in those cases.

5. The case of an $n$-fold multiple root; formal series. We denote the $n$ fold root by $\rho$ and assume $\rho$ not a root of (5); the equation (1) is then satisfied formally by the following $n$ series:

$$
\begin{array}{r}
s_{i}(x)=\rho^{x} e^{\gamma_{i}(n-1) x^{(n-1) / n}+\gamma_{i}(n-2) x(n-2) / n+\cdots+\gamma_{i^{\prime}} x^{\prime 1 / n}} x^{r}\left(1+s_{i}^{\prime} x^{-1 / n}+s_{i}^{\prime \prime} x^{-2 / n}+\cdots\right) \\
(i=1,2, \cdots, n) .
\end{array}
$$

In the calculation of the constants use should be made of the expansion

and of the relations

$$
\begin{aligned}
e^{\gamma(n-k)(x+j)(n-k) / n-\gamma(n-k) x(n-k) / n} & =e^{\gamma(n-k) x(n-k) / n}[(1+j / x)(n-k) / n-1] \\
& =e^{\gamma(n-k) x(n-k) / n[((n-k) / n)(j / x)+\cdots]} \\
& =1+\frac{\gamma^{(n-k)}(n-k) j}{n x^{k / n}}+\cdots \\
(j & =1,2, \cdots, n ; k=1,2, \cdots, n-1)
\end{aligned}
$$

$n^{k-1}-(n-1)^{k}+\frac{(n-1)(n-2)^{k}}{2 !}-\cdots \pm(n-1) 2^{k-1} \mp 1 \begin{cases}=0, & k<n ; \\ >0, & k \geqq n ;\end{cases}$

which are readily established ( $k$ being a positive integer). The $\boldsymbol{\gamma}_{i}^{(n-1)}$ are determined by the equation

$$
-\frac{a_{01} \rho^{n}+a_{11} \rho^{n-1}+\cdots+a_{n-1,1} \rho+a_{n 1}}{\left[n^{n-1}-(n-1)^{n}+\frac{(n-1)(n-2)^{n}}{2 !}-\cdots \pm(n-1) 2^{n-1} \mp 1\right] a_{00} \rho^{n}}-\frac{n ! n^{n-1}}{(n-1)^{n}} .
$$

As soon as we fix upon a determination for $\gamma_{i}^{(n-1)}$, the values of the subsequent constants in the $i$ th series (32) are uniquely determined.

For simplicity we shall throughout our work take that determination of $\arg \gamma_{i}^{(n-1)}$ which is positive or zero and less than $2 \pi$; if another determination were selected, the reasoning would proceed in the same manner. $\gamma_{1}^{(n-1)}$ 
will then be chosen, for convenience, to denote that one of the $\gamma_{i}^{(n-1)}$ whose argument is least. We assign subscripts to the other $\gamma_{i}^{(n-1)}$ so that we have*

$$
\begin{array}{ll}
\gamma_{2}^{(-1)}=\omega_{1} \gamma_{1}^{(-1)}, & \gamma_{3}^{(-1)}=\omega_{n-1} \gamma_{1}^{(n-1)}, \\
\gamma_{1}^{(n-1)}=\omega_{2} \gamma_{1}^{(-1)}, & \gamma_{5}^{(-1)}=\omega_{n-2} \gamma_{1}^{(n-1)}, \text { etc. },
\end{array}
$$

where $\omega_{i}(i=1,2, \cdots, n-1)$ are the complex $n$th roots of unity in the order in which one meets them in traversing the unit circle in counter-clockwise direction beginning at 1 .

Let us set

$$
g_{i}(x)=g(x+i-1) \quad(i=1,2, \cdots, n)
$$

and seek to obtain analytic solutions of equation (1) by obtaining them for the system

$$
\begin{aligned}
& g_{i}(x+1)=g_{i+1}(x) \quad(i=1,2, \cdots, n-1), \\
& g_{n}(x+1)=-\frac{a_{n}(x)}{a_{0}(x)} g_{1}(x)-\frac{a_{n-1}(x)}{a_{0}(x)} g_{2}(x)-\cdots-\frac{a_{1}(x)}{a_{0}(x)} g_{n}(x),
\end{aligned}
$$

or in matrix notation,

$$
G(x+1)=A(x) G(x) .
$$

This equation possesses the formal matrix solution

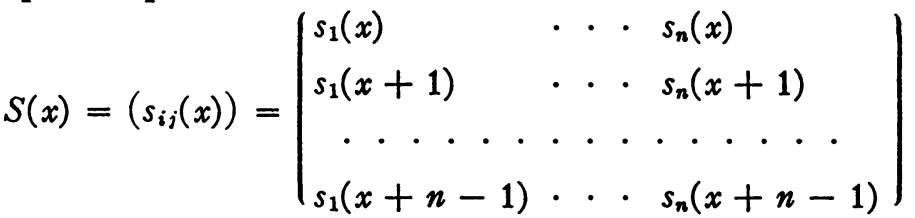

$$
\begin{aligned}
& =\rho^{x} x^{r}\left(\begin{array}{ccc}
e^{L_{1}(x)}\left(1+\frac{s_{1}^{\prime}}{x^{1 / n}}+\cdots\right) & \cdot \cdot e^{L_{n}(x)}\left(1+\frac{s_{n}^{\prime}}{x^{1 / n}}+\cdots\right) \\
e^{L_{1}()}(\rho+\cdots) & \cdot \cdot \cdot e^{L_{n}(x)}(\rho+\cdots) \\
\cdot \cdot \cdot \cdot \cdot \cdot \cdot \cdot \cdot \cdot \cdot \cdot \cdot \cdot \cdot \cdot \cdot \cdot \\
e^{L_{1}(x)}\left(\rho^{n-1}+\cdots\right) & \cdot \cdot \cdot e^{L_{n}(x)}\left(\rho^{n-1}+\cdots\right)
\end{array}\right),
\end{aligned}
$$

* It would seem natural to assign subscripts to the other $\gamma_{i}^{(n-1)}$ so that we should have

$$
\gamma_{i}^{(n-1)}=\omega_{i-1} \gamma_{1}{ }^{(n-1)}
$$

$(i=2,3, \cdots, n)$.

If that assignment were made, we should find that upon replacing $x^{1 / n}$ by $\omega_{1} x^{1 / n}, s_{1}(x)$ would become $s_{n}(x)$ and likewise $s_{i}(x)$ would become $s_{i-1}(x)(i=2,3, \cdots, n)$; indeed we should have

and

$$
\begin{aligned}
\gamma_{i}{ }^{(n-i)}=\omega_{i-j} \gamma_{1}{ }^{(n-i)} & (j=2,3, \cdots, n-1) \\
s_{i}{ }^{(j)}=\omega_{i-j} s_{1}{ }^{(j)} & (j=1,2, \cdots),
\end{aligned}
$$

a set of relations which might be obtained directly by examination of the expressions for the $\gamma$ 's and $s$ 's in terms of the $a$ 's of the coefficient functions (2). It is thus clear that the series in (32) are really the $n$ determinations of a single series. On the other hand the assignment of subscripts in this way would make it necessary for us to order the formal series in the matrix $S(x)$, presently to be introduced, in other than the natural order of the subscripts employed in the series, and this would produce considerable inconvenience in the work to follow. 
where for brevity we have set

$$
L_{i}(x)=\gamma_{j}^{(n-1)} x^{(n-1) / n}+\gamma_{j}^{(n-2)} x^{(n-2) / n}+\cdots+\gamma_{j}^{\prime} x^{1 / n}(j=1,2, \cdots, n),
$$

there being no possibility of ambiguity in this section and the following one if the superscript $(n)$ is omitted from $L_{j}(x)$. Our proof of the existence of analytic solutions of (35) will be based upon modifications of the following infinite products, which are symbolic solutions of that equation:

$$
\begin{aligned}
& A(x-1) A(x-2) A(x-3) \cdots \\
& A^{-1}(x) A^{-1}(x+1) A^{-1}(x+2) \cdots
\end{aligned}
$$

The $n$ columns of the matrix $S(x)$ are linearly independent sets of functions, since the determinant of the matrix is

$$
|S(x)|=\rho^{n x} x^{n r-(n-1) / 2}\left(d+d^{\prime} x^{-1 / n}+\cdots\right), d=C\left[\gamma_{!^{(n-1)}}\right]^{n(n-1) / 2} \neq 0,
$$

$C$ being a constant not zero. In general the series in $S(x)$ do not converge, but it will be shown that in general there exist two solutions of the system (35) which are analytic over the entire finite $x$-plane and which are asymptotically represented in a portion of the plane by certain elements of the matrix $S(x)$.

6. The case of an $n$-fold multiple root; determinant limits. We have observed that $S(x)$ is a formal matrix solution of (36),

$$
S(x+1)=A(x) S(x) \text {. }
$$

The element in the $i$ th row and $j$ th column of the inverse matrix $S^{-1}(x)$, being the quotient of the cofactor of the element in the $j$ th row and $i$ th column of $S(x)$ by the determinant $|S(x)|$ (cf. (40)), is

$$
\bar{s}_{i j}(x)=\rho^{-x} e^{-L_{i}(x)} x^{(n-1) / 2-r}\left(\bar{s}_{i j}+\bar{s}_{i j}^{\prime} x^{-1 / n}+\bar{s}_{i j}^{\prime \prime} x^{-2 / n}+\cdots\right) .
$$

Let $T(x)$ denote the matrix obtained from $S(x)$ by replacing $s_{i j}(x)$ by $t_{i j}(x)$, where the series in the latter is convergent for $x$ in the neighborhood of $\infty$ and has the same terms as the series in $s_{i j}(x)$ up to and including that in $x^{-(K-1) / n}$. We may then define a matrix $B(x)$ by the relation

$$
T(x+1)=B(x) T(x) .
$$

$B(x)$ is a matrix of functions $b_{i j}(x)$ each of which has an expansion in powers of $x^{-1 / n}$ that agrees precisely with $a_{i j}(x)$ (the element in the $i$ th row and $j$ th column of $A(x))$ up to and including the term in $x^{-(K-1) / n}$. This follows upon comparing

$$
B(x)=T(x+1) T^{-1}(x) \quad \text { and } \quad A(x)=S(x+1) S^{-1}(x) ;
$$


for $T(x+1)$ is the same as $S(x+1)$ up to and including the term in $x^{-(K-1) / n}$ and similarly for $T^{-1}(x)$ and $S^{-1}(x)$, because $\bar{t}_{i j}(x)$ of $T^{-1}(x)$ is given by (41) up to and including turms of degree $K-1$ in $x^{-1 / n}$. We therefore have

$$
A(x)=B(x)+M(x)
$$

where $M(x)$ is a matrix of power series in $x^{-1 / n}$ whose lowest degree terms are of degree $K$ or higher and which converge in the vicinity of $\infty$. Then $N(x)$, defined by

$$
M(x)=B(x) N(x),
$$

is a matrix of power series in $x^{-1 / n}$ of the same type as those in $M(x)$. Hence we have

$$
A(x)=B(x)\left[I+x^{-K / n} C(x)\right]
$$

in which $I$ is the unit matrix and $C(x)$ is a matrix of power series in $x^{-1 / n}$ convergent for $|x|>R$.

We shall understand the definition* of asymptotic representation of a function with respect to $x$ or $v\left(x=u+(-1)^{1 / 2} v\right)$ by a power series in $x^{-1}$ to be modified in the natural manner to define representation by a series of the type of those in (32).

For convenience in stating the following theorem let us denote by $\phi$ the principal determination of

$$
\arg \left[\gamma_{1}(-1)\right]^{n}
$$

for the present we assume

$$
0<\phi<\pi .
$$

Theorem A. Form the product of matrices

$$
P_{m}(x)=A(x-1) A(x-2) \cdots A(x-m) T(x-m) .
$$

If a suitable determination of $x^{1 / n}$ be selected and $m$ be allowed to become infinite, each $\lambda$-rowed determinant formed from the first $\lambda$ columns $(\lambda=1,2, \cdots$, $n$ ) of $P_{m}(x)$ converges, for $K$ sufficiently large, to a definite limit function $u_{i j} \ldots l(x)$, independent of $K$. These limit functions are analytic over the entire finite plane except at points which are singularities of $A(x-1)$ or are congruent on the right $\dagger$ to them. For odd [even] values of $\lambda$ less than $n$ the limit functions

- Cf. Birkhoff, loc. cit., p. 248.

$\dagger$ The points $x+1, x+2, x+3, \cdots[x-1, x-2, x-3, \cdots]$ are said to be congruent on the right [left] to the point $x$. 
are asymptotically represented with respect to $x$ in the sector $\pi / 2 \leqq \arg x \leqq \pi$ $+\phi-\epsilon[\phi+\epsilon \leqq \arg x \leqq 3 \pi / 2]$ ( $\epsilon$ being an arbitrarily small positive quantity) by the corresponding determinant $s_{i j} \ldots l(x)$ of $S(x)$. The asymptotic form of $u_{i j} \ldots l(x)$ for $\lambda$ odd [even] and less than $n$ is given by $s_{i j} \ldots l(x)$ with respect to $v$ in the sector $0<\arg x<\pi / 2[3 \pi / 2<\arg x<2 \pi]$. The determinant $\left|P_{m}(x)\right|$ (given by $\lambda=n$ ) is asymptotically represented by the determinant $|S(x)|$ with respect to $x$ in the sector $\pi / 2 \leqq \arg x \leqq 3 \pi / 2$ and with respect to $v$ in the sectors $0<\arg x<\pi$ and $3 \pi / 2<\arg x<2 \pi$.

We may write

$$
P_{m}(x)=T(x) \bar{P}_{m}(x),
$$

where

$$
\begin{array}{r}
\bar{P}_{m}(x)=\left[T^{-1}(x) A(x-1) T(x-1)\right]\left[T^{-1}(x-1) A(x-2) T(x-2)\right] \\
\cdots\left[T^{-1}(x-m+1) A(x-m) T(x-m)\right] .
\end{array}
$$

The elements of $T(x)$ are polynomials or series convergent for $|x|>R$. In order to show that the determinants in $P_{m}(x)$ converge to definite limit functions as $m$ becomes infinite, it will be sufficient to prove that the corresponding determinants in $\bar{P}_{m}(x)$ so converge. We proceed to show this first for $\lambda=1$.

The matrix $\bar{P}_{m}(x)$ is the product of matrices of the type

$$
\begin{aligned}
T^{-1}(x+1) A(x) T(x) & =T^{-1}(x+1) B(x) T(x)+T^{-1}(x+1) B(x) x^{-K / n} C(x) T(x) \\
& =I+x^{-K / n} T^{-1}(x) C(x) T(x),
\end{aligned}
$$

$I$ representing again the unit matrix. The second term is a matrix

$$
\left(x^{-K / n+(n-1) / 2} e^{L_{i}(x)-L_{i}(x)} \lambda_{i j}(x)\right)=\left(\theta_{i j}(x)\right)=\Theta(x),
$$

in which the $\lambda_{i j}(x)$ are power series in $x^{-1 / n}$ convergent in the vicinity of $x=\infty$. We shall now suppose $K$ to be taken $\geqq 2 n+n(n-1) / 2$, so that we have

$$
\frac{K}{n}-\frac{n-1}{2}=d \geqq 2 .
$$

The matrix $\bar{P}_{m}(x)$ may now be written

$$
\begin{gathered}
{[I+\Theta(x-1)][I+\Theta(x-2)] \cdots[I+\Theta(x-m)]} \\
=I+\sum_{k_{1}=1}^{m} \Theta\left(x-k_{1}\right)+\sum_{k_{1}=1}^{m-1} \sum_{k_{2}=k_{1}+1}^{m} \Theta\left(x-k_{1}\right) \Theta\left(x-k_{2}\right)+\cdots
\end{gathered}
$$


The $i$ th element $(i=1,2, \cdots, n)$ in the first column is

$$
\begin{aligned}
\bar{p}_{m: i 1}(x)= & \delta_{i 1}+\sum_{k_{1}=1}^{m} \theta_{i 1}\left(x-k_{1}\right) \\
& +\sum_{k_{1}=1}^{m-1} \sum_{k_{2}-k_{1}+1}^{m}\left[\sum_{\tau=1}^{n} \theta_{i r}\left(x-k_{1}\right) \theta_{r 1}\left(x-k_{2}\right)\right]+\cdots \\
& =\delta_{i 1}+\sum_{k_{1}=1}^{m} \frac{\lambda_{i 1}\left(x-k_{1}\right)}{\left(x-k_{1}\right)^{d}} e^{L_{1}\left(x-k_{1}\right)-L_{i}\left(x-k_{1}\right)} \\
+\sum_{k_{1}=1}^{m-1} \sum_{k_{2}=k_{1}+1}^{m} & {\left[\sum_{\tau=1}^{n} \frac{\lambda_{i \tau}\left(x-k_{1}\right) \lambda_{r 1}\left(x-k_{2}\right)}{\left(x-k_{1}\right)^{d}\left(x-k_{2}\right)^{d}} e^{L_{\tau}\left(x-k_{1}\right)-L_{i}\left(x-k_{1}\right)+L_{1}\left(x-k_{2}\right)-L_{\tau}\left(x-k_{2}\right)}\right]+\cdots }
\end{aligned}
$$

But the $\lambda_{i j}(x)(i, j=1,2, \cdots, n)$ are bounded in the neighborhood of $\infty$; i.e., $\left|\lambda_{i j}(x)\right|(i, j=1,2, \cdots, n)$ is less than some constant $M$ for $|x|>R$. Therefore, if $x$ lies in the region $D$ of the accompanying figure, so that the points $x-i(i=1,2, \cdots)$ are all exterior to the circle of radius $R$ about the origin, the product of the $\lambda$-functions in the typical element in the $(l+1)$ th term of $(43)$,

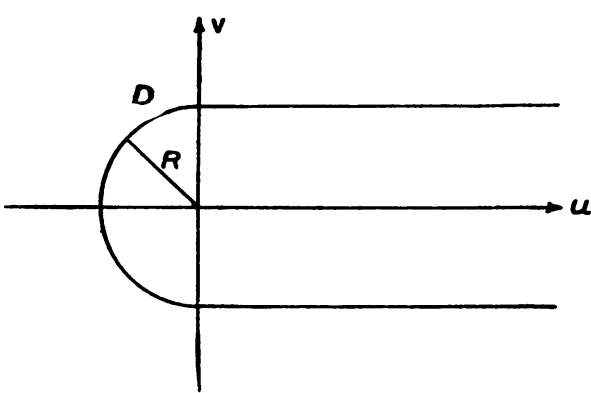

Frg. 2

$$
\begin{gathered}
\frac{\lambda_{i r}\left(x-k_{1}\right) \lambda_{r \sigma}\left(x-k_{2}\right) \cdots \lambda_{\nu 1}\left(x-k_{l}\right)}{\left(x-k_{1}\right)^{d}\left(x-k_{2}\right)^{d} \cdots\left(x-k_{l}\right)^{d}} \\
\cdot e^{L_{r}\left(x-k_{1}\right)-L_{i}\left(x-k_{1}\right)+L_{\sigma}\left(x-k_{2}\right)-L_{\tau}\left(x-k_{2}\right)+\cdots+L_{1}\left(x-k_{l}\right)-L_{\nu}\left(x-k_{l}\right)}
\end{gathered}
$$

is less in absolute value than $M^{l}$. As for the exponential term, that may be written

$$
\begin{array}{r}
e^{L_{1}\left(x-k_{1}\right)-L_{i}\left(x-k_{1}\right)} e^{L_{\gamma}\left(x-k_{1}\right)-L_{\gamma}\left(x-k_{2}\right)-L_{1}\left(x-k_{1}\right)+L_{1}\left(x-k_{2}\right)} \\
\cdot e^{L_{\sigma}\left(x-k_{2}\right)-L_{\sigma}\left(x-k_{q}\right)-L_{1}\left(x-k_{2}\right)+L_{1}\left(x-k_{9}\right)} \\
\cdots e^{L_{y}\left(x-k_{l-1}\right)-L_{y}\left(x-k_{l}\right)-L_{1}\left(x-k_{l-1}\right)+L_{1}\left(x-k_{l}\right)} .
\end{array}
$$

We propose to show that, given any value of $\left[\gamma_{1}^{(n-1)}\right]^{n}$ above the axis of reals, a suitable choice of the determination of $x^{1 / n}$ will make the absolute value of each factor after the first in this product less than or equal to 1 for $x$ in a certain part of the region $D$.

When $\tau$, or $\sigma$, or $\cdots$ is 1 , it is clear that the factor of (45) in which it 
occurs has the value unity. We need consider, therefore, the factors in which the exponent is

$$
\left[L_{j}\left(x-k_{r}\right)-L_{j}\left(x-k_{s}\right)\right]-\left[L_{1}\left(x-k_{r}\right)-L_{1}\left(x-k_{s}\right)\right]\left(\begin{array}{l}
j=2,3, \cdots, n \\
r<s
\end{array}\right) .
$$

Taking into account the relations (34) and (38), we see that this difference may be written in the form

$$
\begin{gathered}
\gamma_{1}^{(n-1)}\left(\omega_{\alpha}-1\right)\left[\left(x-k_{r}\right)^{(n-1) / n}-\left(x-k_{s}\right)^{(n-1) / n}\right] \\
+\left(\gamma_{j}^{(n-2)}-\gamma_{1}^{(n-2)}\right)\left[\left(x-k_{r}\right)^{(n-2) / n}-\left(x-k_{s}\right)^{(n-2) / n}\right] \\
+\cdots+\left(\gamma_{j}^{\prime}-\gamma_{1}^{\prime}\right)\left[\left(x-k_{r}\right)^{1 / n}-\left(x-k_{s}\right)^{1 / n}\right] \\
\alpha=\left\{\begin{array}{l}
\frac{j}{2} \text { for } j \text { even } ; \\
n-\frac{j-1}{2} \text { for } j \text { odd } .
\end{array}\right.
\end{gathered}
$$

If $R$ (cf. Figure 2) is sufficiently large, the first term of this sum is in absolute value the dominating one, whatever determination of $x^{1 / n}$ be selected, for all $x$ in $D$ and for all possible values of $k_{r}$ and $k_{s}$. We now prove this for the case in which $x$ lies in the upper half-plane (including the part of the negative axis of reals in $D$ ) and $\arg x^{1 / n}$ has its smallest positive value; for $x$ in the lower half-plane and for other determinations of $x^{1 / n}$, the situation is wholly similar. The proof is accomplished by showing that, given any constants $C_{p}(\neq 0)$ and $C_{p-1}$ and an arbitrarily large positive number $N$, we can take $R$ so great that the inequality

$$
\left|\frac{C_{p}\left[\left(x-k_{r}\right)^{(n-p) / n}-\left(x-k_{s}\right)^{(n-p) / n}\right]}{C_{p-1}\left[\left(x-k_{r}\right)^{(n-p-1) / n}-\left(x-k_{s}\right)^{(n-p-1) / n}\right]}\right|>N \quad(p=1,2, \cdots, n-2)
$$

is satisfied. The left-hand member may be written

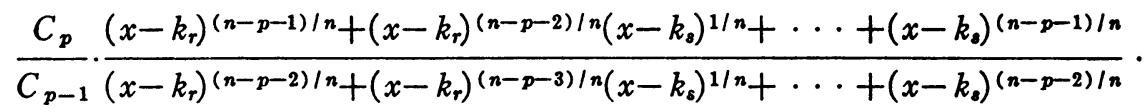

The numerator of the second quotient is to be examined next. The argument of each term in this sum, for any $p$, is positive and $\leqq(n-2) \pi / n$. Hence the argument of the whole sum exclusive of the last term is confined to these same limits, and it follows that the absolute value of the whole sum is*

* If $n \leqq 4$, the factor $\sin (\pi / 2-2 \pi / n)$ occurring here is replaced by 1 , since each term in the sum, for any $p$, has an argument between 0 and $\pi / 2$ (inclusive of the latter). 
$\geqq \sin (\pi / 2-2 \pi / n)$ times the absolute value of that sum with the last term deleted. Thus the absolute value of the expression (48) is

$$
\geqq\left|\frac{C_{p}}{C_{p-1}}\left(x-k_{r}\right)^{1 / n}\right| \sin \left(\frac{\pi}{2}-2 \frac{\pi}{n}\right) .
$$

But $\left|\left(x-k_{r}\right)^{1 / n}\right|>R^{1 / n}$, which we can make as large as we please by taking $R$ sufficiently large.

The results of the preceding paragraph show that if the first term in the sum (47) is kept away from an arbitrarily small sector enclosing the axis of imaginaries, the real part of this first term will be the dominating influence in determining the real part of the function (46). We want the real part of that function to be negative, in order that $e$ to that power shall be in absolute value less than 1 . In other words we desire to have*

$$
\begin{aligned}
\frac{\pi}{2}+\frac{\epsilon}{n} & \leqq \arg \left\{\gamma_{1}^{(n-1)}\left(\omega_{\alpha}-1\right)\left[\left(x-k_{r}\right)^{(n-1) / n}-\left(x-k_{s}\right)^{(n-1) / n}\right]\right\} \\
& \leqq \frac{3 \pi}{2}-\frac{\epsilon}{n},
\end{aligned}
$$

where $\epsilon$ is an arbitrarily small positive number.

By definition of $\phi$ we have

$$
0<\arg \gamma_{1}^{(n-1)}=\frac{\phi}{n}<\frac{\pi}{n} .
$$

It is a simple matter to show that $\arg \left(\omega_{\alpha}-1\right)$ is bounded as follows:

$$
\frac{\pi}{2}+\frac{\pi}{n} \leqq \arg \left(\omega_{\alpha}-1\right) \leqq \frac{3 \pi}{2}-\frac{\pi}{n} \quad(\alpha=1,2, \cdots, n-1) .
$$

Therefore, if condition (49) is to be satisfied, we must have

$$
\text { (50) }-\frac{\pi}{n}-\frac{\phi}{n}+\frac{\epsilon}{n} \leqq \arg \left[\left(x-k_{r}\right)^{(n-1) / n}-\left(x-k_{s}\right)^{(n-1) / n}\right] \leqq \frac{\pi}{n}-\frac{\phi}{n}-\frac{\epsilon}{n} \text {. }
$$

For any particular $x$ the points $x-i(i=1,2,3, \cdots)$ stretch away to the left on the parallel through $x$ to the axis of reals. Such a parallel corresponds to a curve of hyperbolic type (an actual hyperbola if $n=2$ ) in the $x^{1 / n}$-plane, as indicated in Figure 3. $C_{1}$ is the correspondent (first determination) of a parallel in the upper half of the $x$-plane; $C_{2}$ of one in the lower half. Using a second plane for $x^{(n-1) / n}, C_{1}^{\prime}$ and $C_{2}^{\prime}$ (Figure 4) are the transforms

\footnotetext{
* Here and in the next few pages we shall understand the principal value of the argument to be meant unless the contrary is specified.
} 
of $C_{1}$ and $C_{2}$. The vectors are drawn to indicate the value of the arguments of the differences $\left(x-k_{r}\right)^{(n-1) / n}-\left(x-k_{s}\right)^{(n-1) / n}$. From the fact that the mapping is conformal it follows readily that if we have

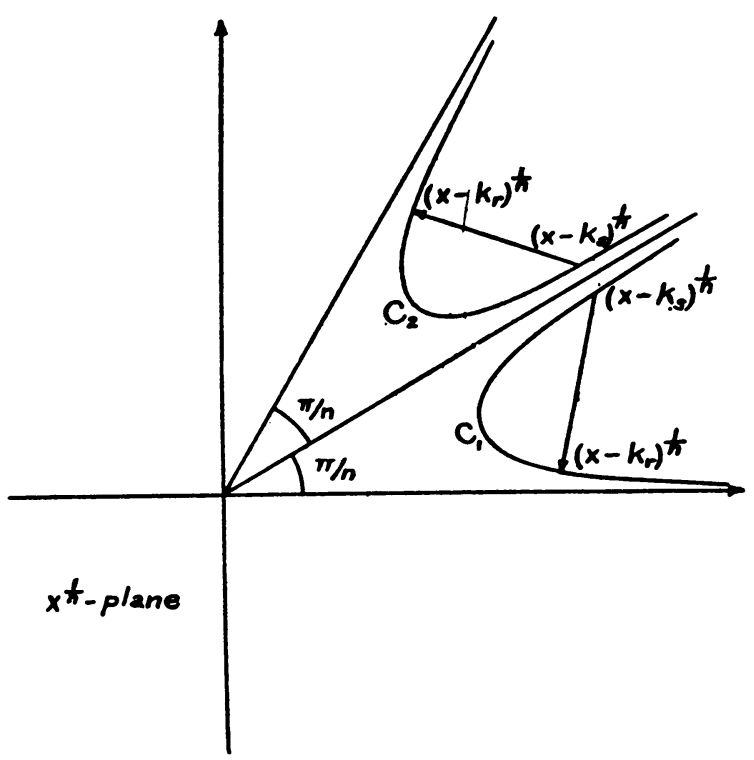

FIG. 3

$$
0 \leqq \arg x \leqq \pi+\phi-\epsilon
$$

and if we select the first determination* for $x^{1 / n}$, the relation (50) will be satisfied. The part of the region $D$ which is also in the sector (51) will hereafter be spoken of as the region $D^{\prime}$.

Returning now to the first factor in (45), we see that it is less in absolute value than $e^{L_{1}(x)-L_{i}(x)}$, since the ratio

$$
\frac{e^{L_{1}\left(x-k_{1}\right)-L_{i}\left(x-k_{1}\right)}}{e^{L_{1}(x)-L_{i}(x)}}=e^{L_{i}(x)-L_{i}\left(x-k_{1}\right)-L_{1}(x)+L_{1}\left(x-k_{1}\right)}
$$

is of the same type as the factors in (45) after the first, and therefore in absolute value less than unity for all $x$ in $D^{\prime}$ and for $k_{1}=1,2,3, \ldots$.

We conclude that for $x$ in $D^{\prime}$ the typical element (44) is less in absolute value than

$$
\frac{M^{l}}{\left(x-k_{1}\right)^{d}\left(x-k_{2}\right)^{d} \cdots\left(x-k_{l}\right)^{d}} e^{L_{1}(x)-L_{i}(x)},
$$

* For brevity we shall speak of the determination of $x^{1 / n}$ for which we have $0 \leqq$ arg $x^{1 / n}<2 \pi / n$ as the first determination, of the determination for which we have $2 \pi / n \leqq \arg x^{1 / n}<4 \pi / n$ as the second determination, etc. 
and hence that the sum (43) is dominated by the sum

$$
\begin{aligned}
\delta_{i 1} & +\left|e^{L_{1}(x)-L_{i}(x)}\right| \sum_{k_{1}=1}^{m} \frac{M}{\left|x-k_{1}\right|^{d}} \\
& +\left|e^{L_{1}(x)-L_{i}(x)}\right| \sum_{k_{1}=1}^{m-1} \sum_{k_{2}=k_{1}+1}^{m} \frac{n M^{2}}{\left|x-k_{1}\right|^{d}\left|x-k_{2}\right|^{d}}+\cdots
\end{aligned}
$$

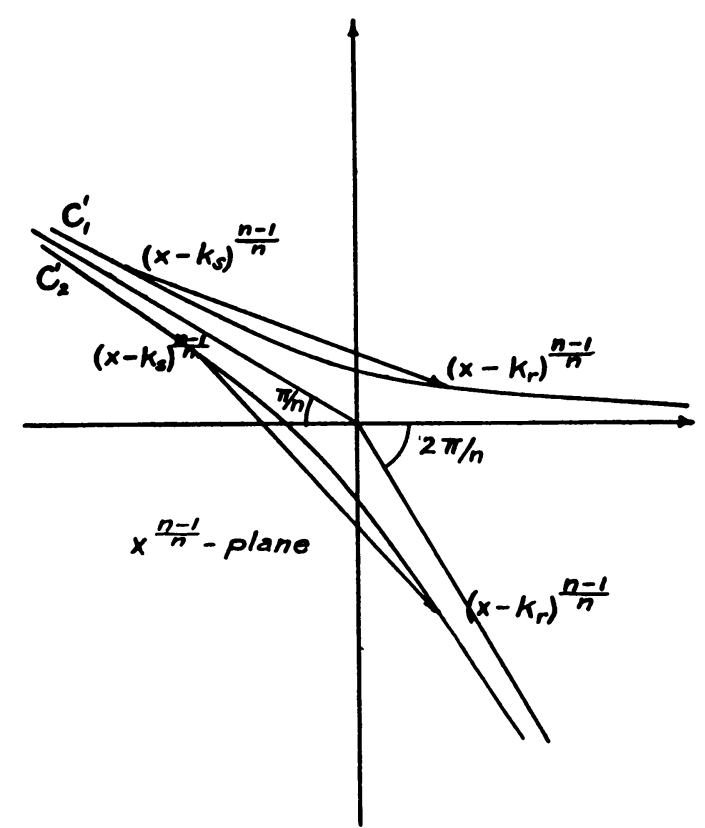

FIG. 4

Allowing $m$ to ${ }^{5}$ become infinite, (43) becomes a multiple series and the sum of terms in (52) after the first approaches the limit

$$
\frac{1}{n}\left|e^{L_{1}(x)-L_{i}(x)}\right|\left\{\left[\left(1+\frac{M}{|x-1|^{d}}\right)\left(1+\frac{M}{|x-2|^{d}}\right) \cdot \cdot\right]-1\right\} .
$$

It is thus clear that the elements of the first column of $\bar{P}_{m}(x)$, and therefore of $P_{m}(x)$, converge absolutely and uniformly to functions analytic in the vicinity of points in $D^{\prime}$. Moreover, it is possible to write

$$
P_{m}(x)=A(x-1) A(x-2) \cdots A(x-r) P_{m-r}(x-r) ;
$$

for any finite $x$ not in $D^{\prime}$, we may take $r$ so large that the points $x-r-1$, $x-r-2, \cdots$ will lie in $D^{\prime}$, and we may carry through for $P_{m-r}(x-r)$ the argument given above for $P_{m}(x)$. Thus the elements of the first column 
of $P_{m}(x)$ converge absolutely and uniformly in the vicinity of any point of the entire finite plane, excepting only points which are singularities of $A(x-1)$ or are congruent on the right to them, and the limit function $u_{i}(x)$ to which $p_{m: i 1}(x)$ converges is analytic throughout the plane with the exceptions noted.

The proof that the limit functions $u_{i}(x)$ are independent of $K$ and that they are asymptotically represented by $s_{i}(x)$ is now carried out as in Birkhoff's paper. The extension of the proof to the cases $\lambda=2,3, \cdots, n$ also involves little modification of his work. For $\lambda=2$ the region of convergence of the elements of the first column of the new difference system is determined by the fact that we have

$$
\frac{\pi}{2}+\frac{2 \pi}{n} \leqq \arg \left(\omega_{\alpha}+\omega_{\beta}-1-\omega_{1}\right) \leqq \frac{3 \pi}{2} \quad(\alpha, \beta=1,2, \cdots, n),
$$

in which $\omega_{n}$ is $1, \alpha$ is $\neq \beta$, and the combination $(\alpha, \beta)$ is distinct from that of $(n, 1)$. Therefore when $\lambda$ is 2 the relation (50) is replaced by

$$
-\frac{2 \pi}{n}-\frac{\phi}{n}+\frac{\epsilon}{n} \leqq \arg \left[\left(x-k_{r}\right)^{(n-1) / n}-\left(x-k_{s}\right)^{(n-1) / n}\right] \leqq-\frac{\phi}{n}-\frac{\epsilon}{n}
$$

and the sector (51) by

$$
\phi+\epsilon \leqq \arg x \leqq 2 \pi .
$$

The case of $\lambda=2$ [1] is typical of that in which $\lambda$ has any even [odd] value $<n$. In the case of $\lambda=n$ no exponential factors (45) occur in the terms of the sum corresponding to (43), since we have

$$
\sum_{j=1}^{n} L_{j}(x)=0 .
$$

Hence convergence can be proved as in the regular case and the asymptotic form $|S(x)|$ is valid for the same region as in that case.

The case of $\left[\gamma_{1}^{(n-1)}\right]^{n}$ in the lower half-plane. If we have

$$
\arg \left[\gamma_{1}^{(n-1)}\right]^{n}=\pi+\phi, \quad 0<\phi<\pi,
$$

the only modification that need be made in Theorem $\mathrm{A}$ is the interchange, for $\lambda$ odd and even and $<n$, of the sectors in which the asymptotic forms of the determinant limits are valid. The determination of $x^{1 / n}$ which must be selected when $\lambda$ is odd is again the first; for $\lambda$ even the second determination must be chosen to secure convergence of the determinants. 
The case of $\left[\gamma_{1}^{(n-1)}\right]^{n}$ real. If $\left[\gamma_{1}^{(n-1)}\right]^{n}$ is negative, the determinant limits exist and are asymptotically represented with respect to $x$ in the sector $\epsilon \leqq \arg x \leqq 2 \pi-\epsilon$. If $\left[\gamma_{1}{ }^{(n-1)}\right]^{n}$ is positive, we cannot be sure of the existence of determinant limits for any determination of $x^{1 / n}$.

In an entirely similar manner we may prove a second theorem in which it is necessary to distinguish between two cases according as $n$ is even or odd.

TheOREM B. Form the product of matrices

$$
P_{m}^{\prime}(x)=A^{-1}(x) A^{-1}(x+1) \cdots A^{-1}(x+m-1) T(x+m) .
$$

If a suitable determination of $x^{1 / n}$ be selected and $m$ allowed to become infinite, each $\lambda$-rowed determinant formed from the last $\lambda$ columns converges, for $K$ sufficiently large, to a definite limit function $v_{i j} \ldots l(x)$, independent of $K$. These limit functions are analytic over the entire finite plane except at points which are singularities of $A^{-1}(x)$ or are congruent on the left to them. For $n$ even and $\lambda$ odd [even] and $<n$ the limit functions are asymptotically represented with respect to $x$ in the sector $-\pi+\phi+\epsilon \leqq \arg x \leqq \pi / 2[-\pi / 2 \leqq \arg x \leqq \phi-\epsilon]$ by the corresponding determinant $s_{i j \ldots l}(x)$ of $S(x)$. The asymptotic form of $v_{i j} \ldots l(x)$ for $n$ even and $\lambda$ odd [even] and $<n$ is given by $s_{i j \ldots l}(x)$ with respect to $v$ in the sector $\pi / 2<\arg x<\pi[-\pi<\arg x<-\pi / 2]$. For $\lambda$ odd the determination of $x^{1 / n}$ chosen is the first for $x$ in the upper half-plane, the nth for $x$ in the lower; for $\lambda$ even the determination chosen is the first in the lower half-plane, the second in the upper. When $n$ is odd the sectors for $\lambda$ odd and even are interchanged. The determinant $\left|P_{m}^{\prime}(x)\right|$ is asymptotically represented by the determinant $|S(x)|$ with respect to $x$ in the sector $-\pi / 2 \leqq \arg x \leqq \pi / 2$ and with respect to $v$ in the sectors $-\pi<\arg x<-\pi / 2$ and $\pi / 2<\arg x<\pi$.

When $\left[\gamma_{1}{ }^{(n-1)}\right]^{n}$ is in the lower half-plane or is real, modifications in Theorem B must be made like those indicated above for Theorem A in these cases.

If $\rho$ is a simple root of the secondary equation (5), results analogous to those of Theorems A, B can be obtained. When $n$ is odd the series which contains no exponential factor

$$
e^{\gamma_{1}^{(n-2)} x^{(n-2) /(n-1)+\cdots}}
$$

(cf. \$1) should be ordered in the $((n+1) / 2)$ th position, the other series being ordered as above. The only change in the results as stated in Theorem $A$ is the interchange of sectors for $\lambda=(n+1) / 2, \cdots, n-1$. When $n$ is even the series from which the exponential factor is missing should be put in the $(n / 2+1)$ th position. The results are the same except that for $\lambda=n / 2$ 
and $\lambda=n / 2+1$ the region of validity of the asymptotic form is somewhat smaller and for $\lambda=n / 2+2, \cdots, n-1$ the sectors are interchanged.

No difficulties stand in the way of proving the existence of solutions associated with the determinant limits. Beyond this point, however, the theory cannot be extended by the use of any of the evaluations of the operator $\Sigma$, either in terms of series or of contour integrals, that have been used up to the present time in the theory of difference equations. In attempting to obtain intermediate or principal solutions on the left, for example, the exponential factors present make it necessary, in order to secure convergence of the series or of the contour integrals, to proceed toward the right, and this cannot be done without going outside the region of validity of the asymptotic form of the functions whose finite integrals we are seeking to determine.

7. The case of an equation of second order with a double root. It seems desirable to call particular attention to the case of the equation of second order whose characteristic equation has a double root, for the reason that in this case the above argument leads to somewhat more inclusive results. We observe first of all that, grouping the results of Theorems A and B for $\lambda=1$, we have one complete set of solutions of the system (35) which are analytic over the entire finite plane and each of which is given asymptotically by the corresponding formal series in a certain portion of the plane.

Secondly it is possible, on the basis of the results of $\S 6$ for $n=2$, to show the existence of intermediate solutions by giving to the operator $\Sigma$ the series evaluation employed by Birkhoff for the same purpose in the regular case. The elements of the first [second] column of the matrix of intermediate solutions on the left [right], together with their asymptotic properties, are provided by Theorem A[B]; the elements of the second [first] column in general differ from those of the regular case in that they are analytic only above or below some parallel to the real axis-whether above or below depends upon the value of $\left[\gamma_{1}^{\prime}\right]^{2}$. If this quantity is represented by a point in the upper or lower half-plane, the region of validity of the elements of the columns in question is respectively above or below a suitable parallel to the axis of reals.

Furthermore, there occurs in the work of $\S 6$ this essential difference when $n$ is $2:$ since $L_{j}(x)$ is a monomial, $\gamma_{j}^{\prime} x^{1 / 2}$, the argument to show the dominance of the leading term of $L_{j}(x)$ drops out and the $\epsilon$ appearing in the inequalities (49), (50), and (51) and in the statement of Theorems A and B is not present. Hence if $\left[\gamma_{1}^{\prime}\right]^{2}$ takes on a negative real value, the $\phi$ of Theorem A becomes $\pi$ and the sector (51) becomes $0 \leqq \arg x \leqq 2 \pi$. The elements of the first column therefore have precisely the same properties 
as in the regular case except that they are analytic in the cut plane, the cut being made from 0 to $\infty$ along the positive axis of reals; intermediate and principal solutions on the left are then readily obtained. Before allowing $\left[\gamma_{1}^{\prime}\right]^{2}$ to assume a positive real value let us observe that in the proof of Theorem $\mathrm{A}$ there is a sector, $-\pi+\phi+\epsilon \leqq \arg x \leqq 0$, which is, so to speak, unused and apparently unusable; for, although inequality (50) will be satisfied if $x-k_{r}$ and $x-k_{s}$ lie in this sector and the $n$th determination of $x^{1 / n}$ is chosen, $k_{r}$ and $k_{s}$ do not remain small and as they increase, the points $x-k_{r}$ and $x-k_{s}$ are sure to move outside the sector. When $n$ is 2 , this sector is $-\pi+\phi \leqq \arg x \leqq 0$. Thus if $\left[\gamma_{1}^{\prime}\right]^{2}$ is a positive real, so that $\phi$ is zero, the sector previously used becomes $0 \leqq \arg x \leqq \pi$ and the unused sector becomes $-\pi \leqq \arg x \leqq 0$, both of which are usable. The determinations of $x^{1 / 2}$ which must be chosen are, however, different for $x$ in the respective half-planesthe first for $x$ in the upper, the second for $x$ in the lower. Hence the limit functions for $x$ in the upper half-plane do not join on analytically along the axis of reals to those for $x$ in the lower half-plane, since the two determinations of $x^{1 / 2}$ join on continuously along the positive axis of reals, which is outside the region $D$. Intermediate, but not principal, solutions can then be obtained as in the regular case. Parallel statements can be made with reference to solutions on the right when $\left[\gamma_{1}^{\prime}\right]^{2}$ is real.

8. Analytic solutions in a large class of irregular cases. We point out briefly the nature of the results which can be obtained by the methods used above in cases in which the characteristic equation (3) has roots of different multiplicities and a full set of formal series can be found. Our considerations will at first be confined to cases in which the roots of (3) are all finite and nonzero, and for the sake of clarity and simplicity we shall begin with a particular case of rather general type. The facts will be stated only for solutions on the left; the situation with respect to solutions on the right is parallel.

Let the roots $\rho_{i}$ of (3) be ordered according to decreasing absolute value and let us suppose that

$$
\begin{aligned}
\rho_{1}, \rho_{2}, \cdots, \rho_{k} & \text { are simple roots; } \\
\rho_{k+1}=\rho_{k+2}=\cdots, \rho_{l} & \text { is a multiple root; } \\
\rho_{l+1}, \rho_{l+2}, \cdots, \rho_{m} & \text { are simple roots; } \\
\rho_{m+1}=\rho_{m+2}=\cdots=\rho_{p} & \text { is a multiple root; and } \\
\rho_{p+1}, \rho_{p+2}, \cdots, \rho_{n} & \text { are simple roots. }
\end{aligned}
$$

Let us assume further that we have 


$$
\left|\rho_{k}\right|>\left|\rho_{k+1}\right|,\left|\rho_{l}\right|>\left|\rho_{l+1}\right|,\left|\rho_{m}\right|>|| \rho_{m+1}\left|, \rho_{p}\right|>\left|\rho_{p+1}\right| .
$$

Then the existence of determinant limits like those of the regular case can be proved for $\lambda=1,2, \cdots, k, l, l+1, \cdots, m, p, p+1, \cdots, n$; for other values of $\lambda$ the determinant limits are analytic except for poles over the entire finite plane, but they are asymptotically represented by the corresponding determinants of $S(x)$ only in regions like $D^{\prime}$ of $\S 6$. The extension to solutions associated with the determinant limits is immediate. For $i=1,2, \cdots, k$ intermediate and principal solutions may be obtained as in the regular case; for $i=k+1$ an intermediate solution can be gotten like those of $\S 7$ if the left-hand member of (5) for $\rho=\rho_{k+1}$ is not real; for $i>k+1$ intermediate solutions can be found only if $\rho_{k+1}$ is a root of multiplicity 2 . In this case intermediate solutions as in $\$ 7$ can be obtained for $i=k+2, \cdots$, $m$; if the left-hand member of (5) for $\rho=\rho_{k+1}$ is a negative real, principal solutions can be obtained for $i=k+1, \cdots, m$. If the left-hand member of (5) both for $\rho=\rho_{k+1}$ and $\rho=\rho_{m+1}$ has its argument in the open interval $(0, \pi)$ or in the open interval $(\pi, 2 \pi)$, an intermediate solution as in $\$ 7$ can be obtained for $i=m+1$. We can go no further than this unless the multiplicity of the root $\rho_{m+1}$ is 2 and the hypothesis of the preceding sentence is also satisfied. If such is the case, intermediate solutions as in $\$ 7$ can be found for $i=m+2, \cdots, n$; when the left-hand member of (5) both for $\rho=\rho_{k+1}$ and $\rho_{m+1}$ is a negative real, principal solutions can be found for $i=m+1, \cdots, n$.

It may be remarked further that if a multiple root and a simple root have the same absolute value, the corresponding formal series should be ordered as are the series in the case referred to at the close of $\S 6$, in which $\rho$ is a simple root of the secondary equation; determinant limits then exist as in that case. If two multiple roots of different multiplicities have the same absolute value, they should be ordered according to decreasing multiplicity; determinant limits exist as in $\S 6$. If two multiple roots have the same absolute value and the same multiplicity, they should be ordered according to decreasing absolute value of the leading $\gamma$ in the corresponding formal series; i.e., the series corresponding to the root $\rho_{i}$ for which the left-hand member of (5) has the larger absolute value should be placed first. The points in the complex plane representing each set of $\gamma$ 's are then located at the vertices of a regular polygon whose center is the origin. If the smaller polygon lies within the larger, the determinant limits for $\lambda$ equal to the value of $i$ which gives the first series of the first set exist as in $\$ 6$, and if the root yielding the first of the two sets is the first multiple root and is not real, an intermediate 
solution can be obtained corresponding to this value of $i$. Whatever the relative size and position of the two polygons may be, the regions of validity of the asymptotic forms of some or all of the determinant limits for $\lambda$ greater than this value of $i$ are further restricted.

The extension of these statements to cases of Class $2, \S 1$, in which the characteristic equations associated with one or more of the segments of $L$ have multiple roots is immediate.

Brown UnIVERstTy,

Providence, R. I. 